\title{
RELAXATION OF AN OPTIMAL DESIGN PROBLEM IN FRACTURE MECHANIC: THE ANTI-PLANE CASE*
}

\author{
Arnaud MÜnCH ${ }^{1}$ And Pablo Pedregal ${ }^{2}$
}

\begin{abstract}
In the framework of the linear fracture theory, a commonly-used tool to describe the smooth evolution of a crack embedded in a bounded domain $\Omega$ is the so-called energy release rate defined as the variation of the mechanical energy with respect to the crack dimension. Precisely, the well-known Griffith's criterion postulates the evolution of the crack if this rate reaches a critical value. In this work, in the anti-plane scalar case, we consider the shape design problem which consists in optimizing the distribution of two materials with different conductivities in $\Omega$ in order to reduce this rate. Since this kind of problem is usually ill-posed, we first derive a relaxation by using the classical non-convex variational method. The computation of the quasi-convex envelope of the cost is performed by using div-curl Young measures, leads to an explicit relaxed formulation of the original problem, and exhibits fine microstructure in the form of first order laminates. Finally, numerical simulations suggest that the optimal distribution permits to reduce significantly the value of the energy release rate.
\end{abstract}

Mathematics Subject Classification. 49J45, 65N30.

Received February 13, 2008. Revised November 15, 2008.

Published online July 2nd, 2009.

\section{IntRoduction - PROBlEM STATEMENT}

We consider a simply connected domain $\Omega$ defined by $\Omega=\Omega_{0} \backslash \gamma$ where $\Omega_{0}$ is a smooth bounded domain of $\mathbb{R}^{2}$ (referred to the orthogonal frame $\left.\left(O ; \boldsymbol{e}_{\mathbf{1}}, \boldsymbol{e}_{\mathbf{2}}\right)\right)$ and $\gamma$ a cut of extremity the point $\boldsymbol{F}$, occupied by two constituent media with constant isotropic conductivity $\alpha$ and $\beta$, respectively, such that $0<\alpha<\beta<\infty$ (see Fig. 1). The overall conductivity in $\Omega$ is denoted by $a_{\mathcal{X}_{\omega}}$ defined by

$$
a_{\mathcal{X}_{\omega}}(\boldsymbol{x})=\alpha \mathcal{X}_{\omega}(\boldsymbol{x})+\beta\left(1-\mathcal{X}_{\omega}(\boldsymbol{x})\right), \quad \boldsymbol{x}=\left(x_{1}, x_{2}\right) \in \Omega
$$

Keywords and phrases. Fracture mechanics, optimal design problem, relaxation, numerical experiments.

* This work has been done while the first author was visiting the Departamento de Matemáticas de la Universidad de CastillaLa Mancha (Ciudad Real). He wishes to express his gratitude to the members of this department for their kind hospitality.

1 UMR CNRS 6623, Université de Franche-Comté, 16 route de Gray, 25030 Besançon Cedex, France.

arnaud.munch@univ-fcomte.fr

On leave to Laboratoire de Mathématiques de Clermont-Ferrand.

Partially supported by grants ANR-05-JC-0182-01 and ANR-07-JC-1832-84.

2 ETSI Industriales, Universidad de Castilla-La Mancha, 13071 Ciudad Real, Spain. Pablo.Pedregal@uclm.es Supported by project MTM2007-62945 from Ministerio de Educación y Ciencia (Spain) and by project PCI08-0084-0424 from JCCM (Castilla-La Mancha). 

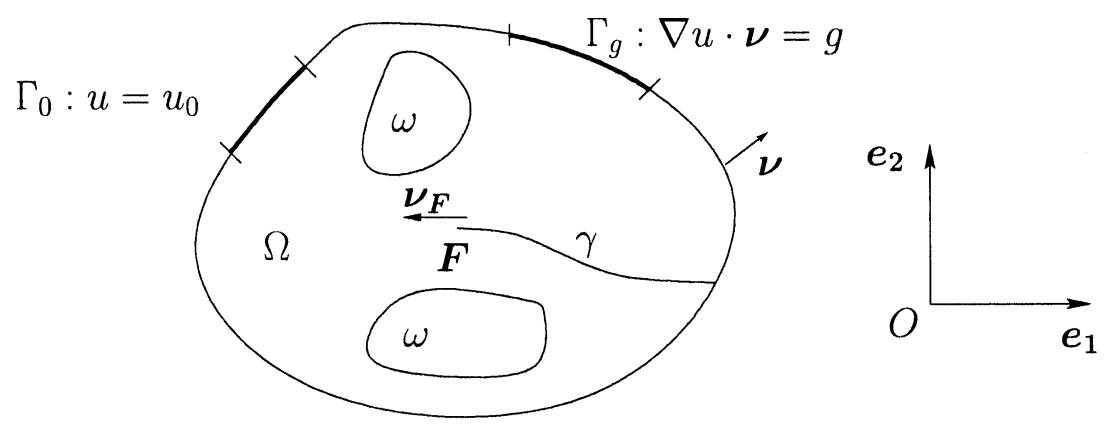

Figure 1. Illustration of problem $(P)$ : optimization of the location of $\omega$ support of the $\alpha$-material in the crack domain $\Omega$.

where $\mathcal{X}_{\omega}$ denotes the characteristic function of any domain $\omega$ strictly included in $\Omega$ (more precisely, $\omega$ is contained in a fixed compact subset $K \subset \subset \Omega)$. We introduce $\Gamma_{0}$ and $\Gamma_{g}$ as two non-empty disjoint parts of $\partial \Omega$ so that $\Gamma_{0} \cap \gamma=\emptyset$ and $\Gamma_{g} \cap \gamma=\emptyset$. For any $u_{0} \in H^{1 / 2}\left(\Gamma_{0}\right)$ and $g \in L^{2}\left(\Gamma_{g}\right)$, we then consider (in a weak sense) the scalar solution $u$ of the following problem

$$
\left\{\begin{array}{lr}
-\operatorname{div}\left(a_{\mathcal{X}_{\omega}}(\boldsymbol{x}) \nabla u\right)=0 & \Omega, \\
u=u_{0} & \Gamma_{0} \subset \partial \Omega, \\
\beta \nabla u \cdot \boldsymbol{\nu}=g & \Gamma_{g} \subset \partial \Omega
\end{array}\right.
$$

where $\boldsymbol{\nu}$ designates the outward unit normal to $\Omega$. The perfect transmission conditions are supposed to hold on $\partial \omega$ between the phase $\alpha$ and $\beta$, i.e. $[u]_{\partial \omega}=0\left([\cdot]_{\partial \omega}\right.$ denotes the jump across $\left.\partial \omega\right)$ and $\alpha \nabla u^{+} \cdot \boldsymbol{\nu}_{\boldsymbol{\omega}}^{+}+$ $\beta \nabla u^{-} \cdot \boldsymbol{\nu}_{\boldsymbol{\omega}}^{-}=0$. The weak solution enjoys the regularity $u \in H^{1}(\Omega)$ (see [15]). Finally, we associate with $u$ the finite energy

$$
E(u, \gamma)=\frac{1}{2} \int_{\Omega} a_{\mathcal{X}_{\omega}}(\boldsymbol{x})|\nabla u|^{2} \mathrm{~d} x-\int_{\Gamma_{g}} g u \mathrm{~d} \sigma .
$$

As it is well-known, the previous system is a simplified scalar version of an elastic structure $S$ occupying the domain $\Omega$, with vanishing displacement on $\Gamma_{0}$, submitted to a normal load $g$ on $\Gamma_{g}$, and containing a crack $\gamma$. This situation is also labeled as the anti-plane case of a linearized elasticity situation. Our motivation in this work is to optimize the distribution of the two materials $\alpha$ and $\beta$ along the structure $S$ in order to prevent, or at least reduce, the growth of the crack point $\boldsymbol{F}$. In this respect, in the framework of Fracture Mechanics (we refer to $[3,18]$ ), a well-known and still widely used growth criterion is due to Griffith [14]. This criterion is related to the so-called energy release, a thermo-dynamical strength named $\mathcal{T}$ (defined as minus the infinitesimal variation of the energy $E$ with respect to variations of $\boldsymbol{F}$ ), and which may be expressed mathematically in term of surface integrals as follows

$$
\mathcal{T}_{\boldsymbol{\psi}}\left(u, \mathcal{X}_{\omega}\right)=\int_{\Omega} a_{\mathcal{X}_{\omega}}(\boldsymbol{x})\left(A_{\boldsymbol{\psi}}(\boldsymbol{x}) \nabla u, \nabla u\right) \mathrm{d} x
$$

provided the function $\boldsymbol{\psi}$ be chosen in a suitable subset of $\left(W^{1, \infty}(\Omega, \mathbb{R})\right)^{2} . A_{\psi}$ is for all $\boldsymbol{x} \in \Omega$ a real $2 \times 2$ matrix, not necessarily positive definite, and (, ) denotes the scalar product in $\mathbb{R}^{2}$ (see Sect. 2). The Griffith's criterion postulates the growth of the point $\boldsymbol{F}$ if $\mathcal{T}_{\psi}\left(u, \mathcal{X}_{\omega}\right)$ reaches a critical positive value experimentally determined. We point out that this criterion, associated with $E$ and recently revisited in [13], is global in contrast with stress criteria such as Von Misses and Tresca criteria. The rate $\mathcal{T}_{\psi}$, which may be seen as a global measure of the singularity of $u$ at the crack tip $\boldsymbol{F}$, is a non-negative but not positive definite function of $u$.

In order to reduce the growth of $\boldsymbol{F}$ due to the load $g$, we therefore consider in this work from a mathematical point of view, the following problem

$$
(P): \quad \inf _{\mathcal{X}_{\omega} \in \mathcal{X}_{L, \mathcal{D}}} \mathcal{T}_{\psi}\left(u, \mathcal{X}_{\omega}\right)
$$


where, for any $L \in(0,1)$ and a suitable compact set $\mathcal{D}$ included in $\bar{\Omega}$ such that $\boldsymbol{F} \in \mathcal{D}$,

$$
\mathcal{X}_{L, \mathcal{D}}=\left\{\mathcal{X} \in L^{\infty}(\Omega,\{0,1\}),\|\mathcal{X}\|_{L^{1}(\Omega)}=L|\Omega|, \mathcal{X}=0 \text { in } \mathcal{D}\right\}
$$

and where $u$ is the solution of (1.2). $(P)$ is a so-called nonlinear optimal design problem associated with a functional which depends quadratically on the gradient of $u$, solution of a partial differential equation. The relation $\|\mathcal{X}\|_{L^{1}(\Omega)}=L|\Omega|$ expresses that the amount of material $\alpha$ to be distributed on $\Omega$ is fixed and equal to $L|\Omega|$ whereas the relation $\mathcal{X}=0$ in $\mathcal{D}$ expresses that the material in $\mathcal{D}$ containing the point $\boldsymbol{F}$ have the characteristic $\beta$. This constraint on the class of characteristic functions will be motivated in Section 2 .

To our knowledge, very few works have investigated the control of the crack growth in this context. We mention two preliminaries notes by Destuynder [8,9]. In [8], the author considers the dynamic wave equation posed on a $2 \mathrm{D}$ cracked domain and defines a growth criterion based on the stress intensity factors. A formulation for the derivative of this criterion is given with respect to a control defined on the boundary of the domain. The reference [9] considers a stationary loaded structure with a crack, and suggests a computational method for a control law which restricts the crack evolution (we refer to [10] for some numerical treatments). In the more recent work [16], an active control strategy is addressed which consists in minimizing the rate $\mathcal{T}_{\psi}$ with respect to the support and amplitude of an additional boundary load. Following this idea, we also mention the note [28] which study the possibility to annihilate the singularity in a cracked domain using additional (singular) boundary loads. Problem $(P)$ is conceptually different and may be qualified as passive control, assuming that the cracked structure is built once for all. On the mathematical viewpoint, this problem is a prototype of ill-posed problem in the sense that the infimum may not be reached in the class of characteristic functions: minimizing sequences oscillate in finer and finer scales. The study of $(P)$ then consists in finding a well-posed relaxation for which there exists an optimal solution. This can be done by mainly two approaches: the Homogenization method (we refer to $[1,34]$ ) and the classical non-convex variational method (we refer to [5,31] and references therein). The well-known application in conductivity is the minimization of the compliance for which the matrix $A_{\psi}$ is simply the identity. The non diagonal and space dependent case provided by the energy release rate $\mathcal{T}_{\boldsymbol{\psi}}$ seems original in this context and requires several new developments. In this work, following some previous works [24-26], we address the relaxation of $(P)$ through the variational method using the class of div-curl Young measures used and analyzed in this context in [33]. The analysis amounts to computing the quasi-convex envelope of the $\operatorname{cost} \mathcal{T}_{\psi}$.

In the spirit of the dynamic material notion introduced recently by Lurie in [19] where material characteristics may vary in time along a spatial domain, the design may be thought of as a dynamical, feed-back control, so that at each position of the growing crack tip $\boldsymbol{F}$, the optimal distribution is adjusted to minimize the energy release rate. This work presents one step in that process and may lead to the optimal design $\left\{\omega_{t}\right\}_{t \in\left(t_{0}, t_{1}\right)}$, assuming that $\boldsymbol{F}$ evolves on the segment $\left[\boldsymbol{F}\left(t_{0}\right), \boldsymbol{F}\left(t_{1}\right)\right] \subset \Omega$ during a time interval $\left[t_{0}, t_{1}\right]$ (this latter being related to the amplitude of the load $g$ ).

The paper is organized as follows. In Section 2, we recall the expression of the energy release rate in terms of a surface integral and some important properties, and then focus on two relevant choices for the matrix $A_{\boldsymbol{\psi}}(\boldsymbol{x})$ : the diagonal, and the non diagonal cases (Rem. 2.1). Then in Section 3, by using the variational approach and Young measures, we determine a full relaxation $(R P)$ of the original problem $(P)$. The analysis is divided into three steps: (i) an equivalent variational reformulation $(V P)$ of $(P)$ (Sect. 3.2); (ii) the computation of a sub-relaxation of $(V P)$ derived from the expression of the poly-convex envelope of $\mathcal{T}_{\psi}$ (Sect. 3.3); (iii) the determination of at least one (div-curl) Young measure for which the lower bound is actually attained. We obtain that both the diagonal and non diagonal cases exhibit first-order laminates (Sect. 3.4). Then, in Section 3.5 by introducing an additional field, we transform the explicit but non standard relaxation $(R P)$ into a new equivalent formulation $(\underline{R P})$ where $u$ appears as a solution of a nonlinear elliptic equation in divergence form (see Eq. (3.75)). This final step then permits to address the numerical approximation and discuss some experiments in Section 4. Some remarks and perspectives conclude this work (Sect. 5). 


\section{Overview ABout the ENERGy RELEASE RATE}

In this section, we recall the definition of the energy release rate and its expression in terms of a surface integral. We use the notation $\psi_{, i}$ for $\partial \psi / \partial x_{i}, i=1,2$, as well as the convention of summation of repeated indices.

We assume that, in the neighborhood of $\boldsymbol{F}$, the crack $\gamma$ is rectilinear and (without loss of generalities) oriented along $\boldsymbol{e}_{\mathbf{1}}$. The energy release rate, designated by $\mathcal{T}$, is formally defined as minus the variation of the elastic energy $E$ with respect to an infinitesimal extension of the crack:

$$
\mathcal{T}=-\lim _{\eta \rightarrow 0} \frac{E\left(u^{\eta}, \gamma^{\eta}\right)-E(u, \gamma)}{\left|\gamma^{\eta}-\gamma\right|}, \quad \eta \in \mathbb{R}_{+}^{*}
$$

where $u^{\eta}$ denotes the solution of (1.2) corresponding to the configuration $\Omega_{0} \backslash \gamma^{\eta}$ obtained after an infinitesimal extension of the point $\boldsymbol{F}$ (in the direction $\boldsymbol{e}_{\mathbf{1}}$ ) parameterized by $\eta$, assuming $u^{0}=u$ and $\gamma^{0}=\gamma \cdot\left|\gamma^{\eta}-\gamma\right|$ denotes the extension length.

The limit $\mathcal{T}$, finite and non negative, may be rigorously expressed in terms of $u$ only. We introduce a velocity field $\boldsymbol{\psi}=\left(\psi_{1}, \psi_{2}\right) \in \boldsymbol{W} \equiv\left\{\boldsymbol{\psi} \in\left(W^{1, \infty}(\Omega, \mathbb{R})\right)^{2}, \boldsymbol{\psi} \cdot \boldsymbol{\nu}=0\right.$ on $\partial \Omega, \boldsymbol{\psi}=0$ on $\left.\Gamma_{g}\right\}$, where $\boldsymbol{\nu}$ designates the unit outward normal to $\Omega$. Let $\eta \in \mathbb{R}_{+}^{*}$ and the transformation $\mathcal{F}^{\eta}: \boldsymbol{x} \rightarrow \boldsymbol{x}+\eta \boldsymbol{\psi}(\boldsymbol{x})$, so that $\mathcal{F}^{\eta}(\boldsymbol{F})=\boldsymbol{F}^{\boldsymbol{\eta}}$ and $\mathcal{F}^{\eta}(\gamma)=\gamma^{\eta}$; we first recall the following definition (see $\left.[3,18]\right)$.

Definition 2.1 (Mathematical definition of the energy release rate). Let $u$ be the solution of (1.2). The derivative of the functional $-E(u, \gamma)$ with respect to a variation of $\gamma$ (more precisely of $\boldsymbol{F}$ ) in the direction $\boldsymbol{\psi}$ is defined as the Fréchet derivative in $\boldsymbol{W}$ at 0 of the application $\eta \rightarrow-E(u,(I d+\eta \boldsymbol{\psi})(\gamma))$, i.e.

$$
-E(u,(I d+\eta \psi)(\gamma))=-E(u, \gamma)-\eta \frac{\partial E(u, \gamma)}{\partial \gamma} \cdot \psi+o(\eta)
$$

In the sequel, we denote this derivative by $\mathcal{T}_{\boldsymbol{\psi}}\left(u, \mathcal{X}_{\omega}\right)$.

The procedure to obtain the explicit expression of $\mathcal{T}_{\boldsymbol{\psi}}$ is technical but by now well-known $($ see $[11,22,23])$. Moreover, since the problem is self-adjoint, the derivative may be expressed only in terms of $u$ as follows.

Lemma 2.1. The first derivative of $-E$ with respect to $\gamma$ in the direction $\boldsymbol{\psi}=\left(\psi_{1}, \psi_{2}\right) \in \boldsymbol{W}$ is given by

$$
\mathcal{T}_{\boldsymbol{\psi}}\left(u, \mathcal{X}_{\omega}\right)=\int_{\Omega} a_{\mathcal{X}_{\omega}}(\boldsymbol{x}) \nabla u \cdot(\nabla \boldsymbol{\psi} \cdot \nabla u) \mathrm{d} x-\frac{1}{2} \int_{\Omega} a_{\mathcal{X}_{\omega}}(\boldsymbol{x})|\nabla u|^{2} \operatorname{div}(\boldsymbol{\psi}) \mathrm{d} x
$$

where $u$ is the solution of (1.2).

Remark that the load $g$ does not occur explicitly in (2.3) since we have assumed for simplicity that $\Gamma_{g} \cap \operatorname{supp} \boldsymbol{\psi}=\emptyset$. Introducing the $2 \times 2$ matrix $A_{\boldsymbol{\psi}}(\boldsymbol{x})$ for all $\boldsymbol{x} \in \Omega$ as follows:

$$
\begin{aligned}
A_{\boldsymbol{\psi}}(\boldsymbol{x}) & =\nabla \boldsymbol{\psi}-\frac{1}{2} \operatorname{div}(\boldsymbol{\psi}) I_{2}=\nabla \boldsymbol{\psi}-\frac{1}{2} \operatorname{Tr}(\nabla \boldsymbol{\psi}) I_{2} \\
& =\frac{1}{2}\left(\begin{array}{cc}
\psi_{1,1}-\psi_{2,2} & 2 \psi_{1,2} \\
2 \psi_{2,1} & \psi_{2,2}-\psi_{1,1}
\end{array}\right)
\end{aligned}
$$

the energy release rate takes the form (1.4).

Moreover, since $\mathcal{T}_{\boldsymbol{\psi}}$ is a shape derivative (with respect to $\boldsymbol{F}$ ), $\mathcal{T}_{\boldsymbol{\psi}}$ should depend on the function $\boldsymbol{\psi} \in \boldsymbol{W}$ only in a neighborhood of the crack tip $\boldsymbol{F}$. This invariance is true for all $\boldsymbol{\psi} \in \boldsymbol{W}$ in the homogeneous case for which $\alpha=\beta$; in our situation, this invariance remains true if the material is homogeneous on the support of the function $\boldsymbol{\psi}$, localized around $\boldsymbol{F}$. We therefore impose that $\operatorname{supp}(\boldsymbol{\psi}) \in \mathcal{D}$, where $\mathcal{D}$ is the set appearing in the definition of the admissible class $\mathcal{X}_{L, \mathcal{D}}($ see $(1.6))$. We then take

$$
\boldsymbol{\psi} \in \boldsymbol{W}_{\mathcal{D}}=\{\boldsymbol{\psi} \in \boldsymbol{W}, \operatorname{supp}(\boldsymbol{\psi}) \subset \mathcal{D}\}
$$


This material assumption then permits to link the derivative $\mathcal{T}_{\boldsymbol{\psi}}$, which is a mathematical quantity defined on $\Omega$, to the thermo-dynamic strength $\mathcal{T}$ (locally defined on $\boldsymbol{F}$ ).

Lemma 2.2 ((local) energy release rate). Let $C(\boldsymbol{F}, r)$ be the circle of center $\boldsymbol{F}$ and radius $r>0, \nu_{\boldsymbol{c}}=\left(\nu_{c, 1}, \nu_{c, 2}\right)$ its outward normal and

$$
\mathcal{T}_{r}\left(u, \mathcal{X}_{\omega}\right)=\frac{1}{2} \int_{C(\boldsymbol{F}, r)} a_{\mathcal{X}_{\omega}}(\boldsymbol{x}) u_{, j} u_{, j} \psi_{k} \nu_{c, k} \mathrm{~d} \sigma-\int_{C(\boldsymbol{F}, r)} a_{\mathcal{X}_{\omega}}(\boldsymbol{x}) u_{, j} u_{, k} \psi_{k} \nu_{c, j} \mathrm{~d} \sigma,
$$

where $u$ is solution of (1.2). The thermo-dynamic strength $\mathcal{T}$ is linked to $\mathcal{T}_{\psi}$ as follows:

$$
\mathcal{T}_{\boldsymbol{\psi}}\left(u, \mathcal{X}_{\omega}\right)=\lim _{r \rightarrow 0} \mathcal{T}_{r}\left(u, \mathcal{X}_{\omega}\right)(\boldsymbol{\psi} \cdot \boldsymbol{\nu})_{\mid \boldsymbol{F}} \equiv \mathcal{T}\left(u, \mathcal{X}_{\omega}\right) \boldsymbol{\psi}(\boldsymbol{F}) \cdot \boldsymbol{\nu}_{\boldsymbol{F}}, \quad \forall \boldsymbol{\psi} \in \boldsymbol{W}_{\mathcal{D}}
$$

where $\boldsymbol{\nu}_{\boldsymbol{F}}=\left(\nu_{F, 1}, \nu_{F, 2}\right)=( \pm 1,0)$ denotes the orientation of the crack $\gamma$ at the point $\boldsymbol{F}$.

It follows from (2.6) that the energy release rate $\mathcal{T}_{\boldsymbol{\psi}}$ is related to the strength $\mathcal{T}$ by

$$
\mathcal{T}\left(u, \mathcal{X}_{\omega}\right)=\mathcal{T}_{\boldsymbol{\psi}}\left(u, \mathcal{X}_{\omega}\right), \quad \forall \boldsymbol{\psi} \in \boldsymbol{W}_{\mathcal{D}} \text { such that } \boldsymbol{\psi}(\boldsymbol{F}) \cdot \boldsymbol{\nu}_{\boldsymbol{F}}= \pm \psi_{1}(\boldsymbol{F})=1 .
$$

As a summary, if the conductivity is constant equal to $\beta$ in $\mathcal{D}$, and if the function $\psi$, which permits to define the virtual crack extension of $\boldsymbol{F}$, belongs to $\boldsymbol{W}_{\mathcal{D}}$ and satisfies $\psi_{1}(\boldsymbol{F})= \pm 1$, then the energy release rate $\mathcal{T}$ may be related to the mathematical quantity $\mathcal{T}_{\psi}$.

\section{Remark 2.1.}

- Since the crack is oriented along the axis $\boldsymbol{e}_{\mathbf{1}}$, a natural choice is $\boldsymbol{\psi}=\left(\psi_{1}, 0\right)$ with $\psi_{1} \nu_{1}=0$ on $\partial \Omega$. In that case, $A_{\psi}$ is simply

$$
A_{\psi}=\frac{1}{2}\left(\begin{array}{cc}
\psi_{1,1} & 2 \psi_{1,2} \\
0 & -\psi_{1,1}
\end{array}\right) .
$$

Moreover, since only the derivative of $\boldsymbol{\psi}$ is involved in $\mathcal{T}_{\boldsymbol{\psi}}$ defined by (2.3), it is more accurate from a numerical point of view to consider a function $\psi_{1}$ which is constant in a neighborhood of $\boldsymbol{F}$. This permits to obtain the strength $\mathcal{T}$ with the relation (2.3) only as a function of the solution $\boldsymbol{u}$ far away from $\boldsymbol{F}$ where it is singular [11]. A simple choice is given by the radial function

$$
\psi_{1}(\boldsymbol{x})=\zeta(\operatorname{dist}(\boldsymbol{x}, \boldsymbol{F})) \nu_{F, 1}, \quad \forall \boldsymbol{x} \in \Omega
$$

defining the function $\zeta \in C^{1}\left(\mathbb{R}^{+} ;[0,1]\right)$ as follows:

$$
\zeta(r)=\left\{\begin{array}{cr}
1 & r \leq r_{1} \\
\frac{\left(r-r_{2}\right)^{2}\left(3 r_{1}-r_{2}-2 r\right)}{\left(r_{1}-r_{2}\right)^{3}} & r_{1} \leq r \leq r_{2} \\
0 & r \geq r_{2}
\end{array}\right.
$$

with $0<r_{1}<r_{2}$ so that $\operatorname{supp}\left(\psi_{1}\right) \subset \mathcal{D}$. Note that this situation, described in Figure 2, leads, for some $\boldsymbol{x} \in \Omega$, to a non-diagonal and non-positive definite matrix $A_{\psi}(\boldsymbol{x})$.

- Let us denote $\left(x_{F, 1}, x_{F, 2}\right)$ the coordinates of $\boldsymbol{F}, \Omega_{\epsilon}=\left\{\boldsymbol{x} \in \Omega,\left|x_{1}-x_{F, 1}\right| \leq \epsilon\right\}$ and $\partial \Omega_{\epsilon}=\{\boldsymbol{x} \epsilon$ $\left.\partial \Omega,\left|x_{1}-x_{F, 1}\right| \leq \epsilon\right\}$. If there exists an $\epsilon>0$ for which $\nu_{1}=0$ on $\partial \Omega_{\epsilon}$ and $\partial \Gamma_{g} \cap \partial \Omega_{\epsilon}=\emptyset$, then one may construct an admissible function $\psi_{1}$ independent of $x_{2}$. It suffices to take $\psi_{1}\left(x_{1}, x_{2}\right)=\zeta\left(x_{1}\right)$

$$
\zeta\left(x_{1}\right)=\left\{\begin{array}{cc}
0 & x_{1} \leq r_{1}, \\
\frac{\left(x_{1}-r_{1}\right)^{2}\left(2 x_{1}+r_{1}-3 r_{2}\right)}{r_{1}-r_{2}} & r_{1} \leq x_{1} \leq r_{2}, \\
1 & r_{2} \leq x_{1} \leq r_{3}, \\
\frac{\left(x_{1}-r_{4}\right)^{2}\left(2 x_{1}+r_{4}-3 r_{3}\right)}{r_{4}-r_{3}} & r_{3} \leq x_{1} \leq r_{4}, \\
0 & x_{1} \geq r_{4},
\end{array}\right.
$$




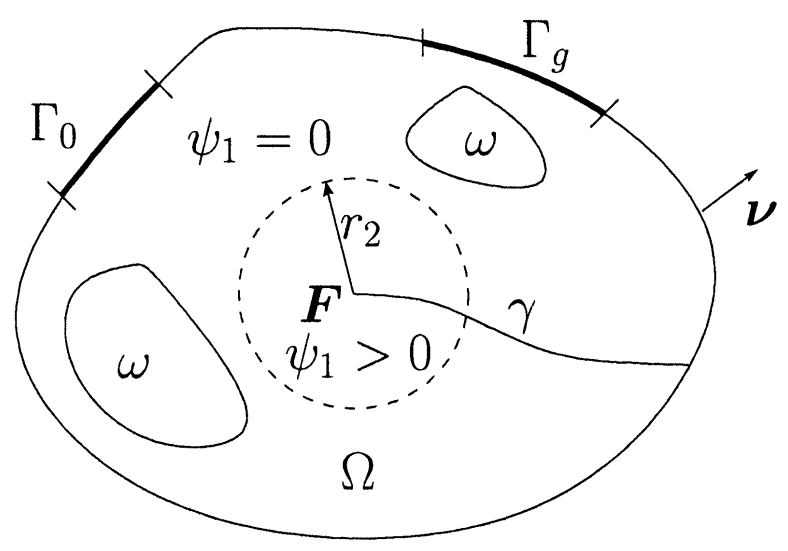

Figure 2. Choice of a radial function $\psi_{1}(\boldsymbol{x})$ leading to a non diagonal matrix $A_{\psi}$.

with

$$
r_{1}=x_{F, 1}-\frac{2 \epsilon}{3}, \quad r_{2}=x_{F, 1}-\frac{\epsilon}{3}, \quad r_{3}=x_{F, 1}+\frac{\epsilon}{3}, \quad r_{4}=x_{F, 1}+\frac{2 \epsilon}{3} .
$$

In this case, $\psi_{1,2}=0$ and the matrix $A_{\psi}$ defined in (2.8) is simply diagonal for all $\boldsymbol{x} \in \Omega$. However, this choice, described in Figure 3, forces the material to be constant in the whole vertical strip $\mathcal{D} \equiv\{\boldsymbol{x} \in \Omega$, $\left.\left|x_{1}-x_{F, 1}\right| \leq r_{4}\right\}$ which is more restrictive than the previous situation.

- One may also construct, for any domain, a function $\boldsymbol{\psi}=\left(\psi_{1}, \psi_{2}\right)$ such that $\psi_{1,2}=\psi_{2,1}$. In this more general case, the matrix $A_{\psi}$ defined by (2.4) is symmetric. It suffices to take $\psi_{1}$ given by $(2.10)$ and $\psi_{2}\left(x_{1}, x_{2}\right)=\int_{0}^{x_{1}} \psi_{1,2}\left(s, x_{2}\right) \mathrm{d} s$. Since $\psi_{1}$ is radial, $\psi_{1,2}=0$ on $\Gamma_{x_{F}}=\left\{\left(x_{1}, x_{2}\right) \in \Omega, x_{1}=x_{F, 1}\right\}$. Therefore, $\psi_{2}=0$ on $\Gamma_{x_{F}}$, and the virtual extension $\boldsymbol{F}^{\boldsymbol{\eta}}=\boldsymbol{F}+\eta \boldsymbol{\psi}(\boldsymbol{F})$ remains on $\Gamma_{x_{F}}$ for all $\eta>0$ small.

- From the definition, $u=0$ implies $\mathcal{T}_{\psi}\left(u, \mathcal{X}_{\omega}\right)=0$. The converse is not true, as a consequence of the non-positive definiteness of the matrix $A_{\psi}$.

\section{Relaxation of $(P)$}

Problem $(P)$, which involves a functional depending on the gradient $\nabla u$, typically lacks optimal solution which means that the infimum may only be achieved by a sequence of more and more intricate subset $\omega_{n}$ of $\Omega$ (see for instance $[27,34]$ ). The goal of this section is to perform a relaxation of problem $(P)$. It consists in looking for another minimization problem $(R P)$ for which there does exist an optimal solution, this minimum has the same value of the infimum of $(P)$, and more importantly, the optimal solution of the relaxed problem encodes the information about (some) minimizing sequence for the original problem. Following the procedure described in [33], a relaxed problem may be obtained by using Young measures generated by sequence of pairs $\left\{G_{n}, H_{n}\right\}$, associated with the design $\mathcal{X}_{\omega_{n}}$ admissible for $(P)$, for which we have the information that the divergence of the first component vanishes while the second component is a gradient. Such class of Young measures, the so-called div-curl Young measures, has been explicitly introduced and studied in [33].

\subsection{Some properties of the class of div-curl Young measures}

As already indicated, all of the material in this section is taken from [33]. It is included in this section for the convenience of the reader. 


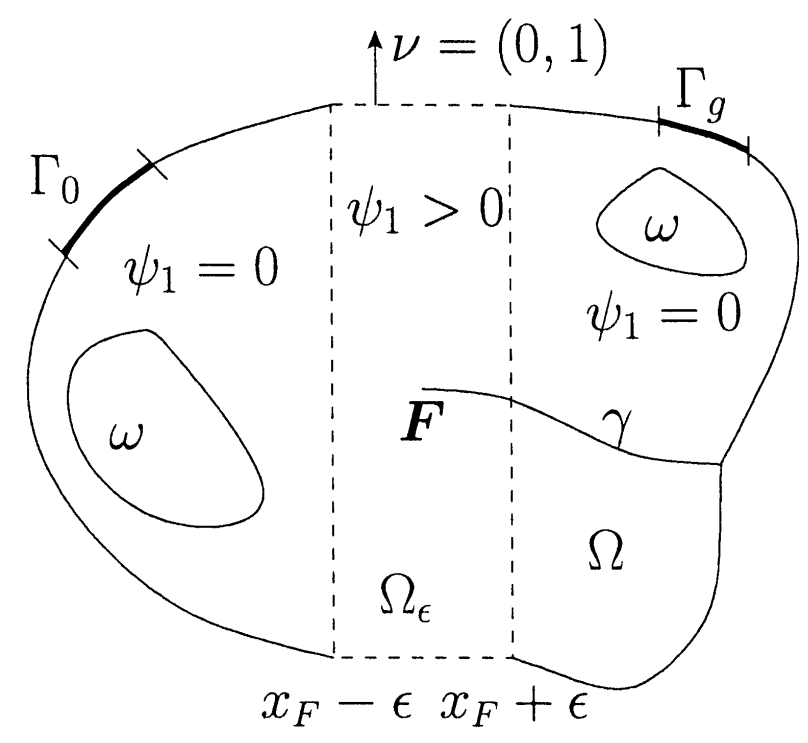

FiguRE 3 . Choice of a function $\psi_{1}(\boldsymbol{x})=\psi_{1}\left(x_{1}\right) \mathcal{X}_{\Omega_{\epsilon}}$ leading to a diagonal matrix $A_{\psi}$ assuming the existence of a domain $\Omega_{\epsilon}$.

Suppose that $\left(\mathcal{X}_{\omega_{n}}\right)_{(n>0)}$ is a minimizing sequence for $(P)$ and let $u_{n}$ be its corresponding sequence of solutions. Consider the two sequences of vectors

$$
G_{n}(\boldsymbol{x})=\left(\alpha \mathcal{X}_{\omega_{n}}+\beta\left(1-\mathcal{X}_{\omega_{n}}\right)\right) \nabla u_{n}(\boldsymbol{x}), \quad H_{n}(\boldsymbol{x})=\nabla u_{n}(\boldsymbol{x}) .
$$

Since both sequences are uniformly bounded in $\left(L^{2}(\Omega)\right)^{2}$, we may associate with (a subsequence of) the pair $\left(G_{n}, H_{n}\right)$ a family of parameterized measures $\nu=\left\{\nu_{x}\right\}_{x \in \Omega}$. Since the pair satisfies $\operatorname{div} G_{n}=0$ and $\operatorname{curl} H_{n}=0$ weakly in $\Omega$, the measure $\nu$ is called a div-curl Young measure. More precisely, we have the following definition.

Definition 3.1. A family of probability measures $\nu=\left\{\nu_{x}\right\}_{x \in \Omega}$ is called a $\left(L^{2}\right.$-) div-curl Young measure if there exists a sequence of pairs of vector fields $G_{n}$ in $L^{2}\left(\Omega ; \mathbb{R}^{m}\right)$, and $u_{n}$ in $H^{1}\left(\Omega ; \mathbb{R}^{m}\right)$, such that

$$
\operatorname{div} G_{n} \rightarrow 0 \text { in } H^{-1}\left(\Omega ; \mathbb{R}^{m}\right), \quad\left\{\left|G_{n}\right|^{2}\right\},\left\{\left|\nabla u_{n}\right|^{2}\right\} \text { are equiintegrable in } \Omega,
$$

and the Young measure associated with $\left\{\left(G_{n}, \nabla u_{n}\right)\right\}$ is $\nu$.

Notice how the equi-integrability ingredient comes directly from standard properties for elliptic equations.

As a consequence of the div-curl lemma, such class of measures enjoys the following commutation property:

$$
\int_{\mathbb{R}^{2} \times \mathbb{R}^{2}} \rho \cdot \lambda \mathrm{d} \nu_{x}(\rho, \lambda)=\int_{\mathbb{R}^{2}} \rho \mathrm{d} \nu_{x}^{(1)}(\rho) \int_{\mathbb{R}^{2}} \lambda \mathrm{d} \nu_{x}^{(2)}(\lambda)
$$

where $\nu_{x}^{(i)}, i=1,2$, are the marginal on the two components, respectively.

In our situation, since $G_{n}$ comes from the state equation, each individual $\nu_{x}$ is supported in the union of the two linear manifolds

$$
\Lambda_{\gamma}=\left\{(\lambda, \rho) \in \mathbb{R}^{2} \times \mathbb{R}^{2}: \rho=\gamma \lambda\right\}, \quad \gamma=\alpha, \beta
$$

so that $\operatorname{supp}\left(\nu_{x}\right) \subset \Lambda_{\alpha} \cup \Lambda_{\beta}$. Because of this fact on the support, the measure $\nu_{x}$ may be decomposed as

$$
\nu_{x}=s(\boldsymbol{x}) \nu_{x, \alpha}+(1-s(\boldsymbol{x})) \nu_{x, \beta}
$$


with $\operatorname{supp}\left(\nu_{x, \gamma}\right) \subset \Lambda_{\gamma}$ and $s(\boldsymbol{x}) \in[0,1]$, the weak $-\star$ limit in $L^{\infty}(\Omega)$ of a subsequence of $\mathcal{X}_{\omega_{n}}$. The meaning of the manifolds $\Lambda_{\gamma}, \gamma=\alpha, \beta$, and, in particular, of the dummy variables $(\rho, \lambda)$, follows from the fact that the measure $\nu_{x}$ gives the limiting probability distribution as $n$ goes to infinity of the values of $\left(G_{n}, H_{n}\right)$ near the point $\boldsymbol{x}$. Then, by the fundamental property of Young measures (see [30], Thm. 6.2), we may represent the limit of the cost associated with $\mathcal{X}_{\omega_{n}}$ through the measure $\nu$. Let us use the notation : to indicate full contraction of the scalar product of two matrices, so that for instance the relation $\left(A_{\psi} \nabla u, \nabla u\right)=A_{\psi}:\left(\nabla u \nabla u^{T}\right)$ holds where $\nabla u^{T}$ denotes the transpose of the vector $\nabla u$. Then, the limit of the cost is

$$
\lim _{n \rightarrow \infty} \mathcal{T}_{\psi}\left(u_{n}, \mathcal{X}_{\omega_{n}}\right)=\int_{\Omega}\left[\alpha s(\boldsymbol{x}) A_{\psi}(\boldsymbol{x}): \int_{\mathbb{R}^{2}} \lambda \lambda^{T} \mathrm{~d} \nu_{x, \alpha}^{(1)}(\lambda)+\beta(1-s(\boldsymbol{x})) A_{\boldsymbol{\psi}}(\boldsymbol{x}): \int_{\mathbb{R}^{2}} \lambda \lambda^{T} \mathrm{~d} \nu_{x, \beta}^{(1)}(\lambda)\right] \mathrm{d} x
$$

where $\nu_{x, \gamma}^{(1)}, \gamma=\alpha, \beta$, designates the projection of $\nu_{x, \gamma}$ onto the first copy of $\mathbb{R}^{2}$. Therefore, with each minimizing sequence of $(P)$, we associate an optimal div-curl Young measure. In this sense, optimizing with respect to $\mathcal{X}_{\omega_{n}}$ is equivalent to optimizing with respect to $\nu$.

In this process (in the relaxed formulation), the following characterization of this class of Young measures is used in a fundamental way.

Lemma 3.1. A parameterized measure $\nu=\left\{\nu_{x}\right\}_{x \in \Omega}$ is a div-curl Young measure if and only if:

- For a.e. $\boldsymbol{x} \in \Omega$, each individual $\nu_{x}$ is a homogeneous, div-curl Young measure by itself;

- For the first-moment of $\nu_{x}$ as a function of $x$, we have

$$
\operatorname{div}\left(\int_{\mathbb{R}^{2}} \rho \mathrm{d} \nu_{x}^{(1)}(\rho)\right)=0, \quad \operatorname{curl}\left(\int_{\mathbb{R}^{2}} \lambda \mathrm{d} \nu_{x}^{(2)}(\lambda)\right)=0 \text { in } \Omega .
$$

Finally, the following result refers to a specific, general way of constructing explicitly some div-curl Young measures. This is the analogue of laminates for gradient Young measures.

Lemma 3.2. Suppose that $\rho_{i}, \lambda_{i}, i=1,2$, are four vectors in $\mathbb{R}^{2}$ such that

$$
\left(\rho_{2}-\rho_{1}\right) \cdot\left(\lambda_{2}-\lambda_{1}\right)=0 .
$$

Then the probability measure

is a div-curl Young measure for all $t \in[0,1]$.

$$
\mu=t \delta_{\left(\rho_{1}, \lambda_{1}\right)}+(1-t) \delta_{\left(\rho_{2}, \lambda_{2}\right)}
$$

\subsection{Variational reformulation}

We now proceed to the analysis of problem $(P)$ in a similar fashion as in [33]. We first put $(P)$ in an equivalent variational setting. We introduce the two functions

$$
W(\boldsymbol{x}, \rho, \lambda)=\left\{\begin{array}{lrr}
\alpha A_{\boldsymbol{\psi}}(\boldsymbol{x}): \lambda \lambda^{T} & \text { if } & (\rho, \lambda) \in \Lambda_{\alpha} \\
\beta A_{\psi}(\boldsymbol{x}): \lambda \lambda^{T} & \text { if } & (\rho, \lambda) \in \Lambda_{\beta} \\
+\infty & & \text { else }
\end{array}\right.
$$

and

$$
V(\rho, \lambda)=\left\{\begin{array}{lrr}
1 & \text { if } \quad(\rho, \lambda) \in \Lambda_{\alpha} \\
0 & \text { if } \quad(\rho, \lambda) \in \Lambda_{\beta} \\
+\infty & & \text { else }
\end{array}\right.
$$

and point out that $W$ and $V$ are not continuous with respect to $(\rho, \lambda)$. Then we check that $(P)$ is equivalent to the following new problem

$$
(V P): \quad \inf _{G, u} \int_{\Omega} W(\boldsymbol{x}, G(\boldsymbol{x}), \nabla u(\boldsymbol{x})) \mathrm{d} x
$$


subject to

$$
\left\{\begin{array}{l}
G \in L^{2}\left(\Omega ; \mathbb{R}^{2}\right), \quad u \in H^{1}(\Omega ; \mathbb{R}) \\
\operatorname{div} G=0 \text { in } H^{-1}(\Omega), \quad G(\boldsymbol{x})=\beta \nabla u(\boldsymbol{x}) \text { in } \mathcal{D} \\
u=u_{0} \text { on } \Gamma_{0}, \quad \beta \nabla u \cdot \boldsymbol{\nu}=g \text { on } \Gamma_{g} \subset \partial \Omega \backslash\left(\gamma \cup \Gamma_{0}\right) \\
\int_{\Omega} V(G(\boldsymbol{x}), \nabla u(\boldsymbol{x})) \mathrm{d} x=L|\Omega|
\end{array}\right.
$$

This equivalent formulation suffers from the same troubles as the initial problem, so that it is in need of relaxation. The crucial step is the computation of the constrained quasi-convexification $C Q W$ of the density $W$ leading to a relaxation $(R P)$ of $(V P)$ (and thus of $(P))$ :

$$
(R P): \quad \min _{s, G, u} \int_{\Omega} C Q W(\boldsymbol{x}, s(\boldsymbol{x}), G(\boldsymbol{x}), \nabla u(\boldsymbol{x})) \mathrm{d} x
$$

for $s \in L^{\infty}(\Omega,[0,1])$ satisfying $s=0$ in $\mathcal{D} \cup \partial \Omega$, and subject to (3.12), but replacing the integral constraint involving $V$ by $\int_{\Omega} s(\boldsymbol{x}) \mathrm{d} x=L|\Omega|$. In the sequel, we note

$$
s \in S_{L, \mathcal{D}} \equiv\left\{s \in L^{\infty}(\Omega,[0,1]),\|s\|_{L^{1}(\Omega)}=L|\Omega|, s=0 \text { in } \mathcal{D} \cup \partial \Omega\right\}
$$

The constrained quasi-convex density $C Q W$ is computed by solving the problem in measures:

$$
C Q W(\boldsymbol{x}, s(\boldsymbol{x}), G(\boldsymbol{x}), \nabla u(\boldsymbol{x}))=\inf _{\nu}\left\{\alpha s(\boldsymbol{x}) A_{\boldsymbol{\psi}}(\boldsymbol{x}): \int_{\mathbb{R}^{2}} \lambda \lambda^{T} \mathrm{~d} \nu_{x, \alpha}^{(1)}(\lambda)+\beta(1-s(\boldsymbol{x})) A_{\boldsymbol{\psi}}(\boldsymbol{x}): \int_{\mathbb{R}^{2}} \lambda \lambda^{T} \mathrm{~d} \nu_{\boldsymbol{x}, \beta}^{(1)}(\lambda)\right\}
$$

for any measure $\nu$ subject to

$$
\left\{\begin{array}{l}
\nu=\left\{\nu_{x}\right\}_{x \in \Omega}, \quad \nu_{x}=s(\boldsymbol{x}) \nu_{\boldsymbol{x}, \alpha}+(1-s(\boldsymbol{x})) \nu_{\boldsymbol{x}, \beta}, \quad \operatorname{supp}\left(\nu_{\boldsymbol{x}, \gamma}\right) \subset \Lambda_{\gamma}, \\
\nu \text { is div-curl Young measure (satisfying }(3.2)), \\
G(\boldsymbol{x})=\int_{\mathbb{R}^{2}} \rho \mathrm{d} \nu_{x}(\lambda, \rho), \quad \operatorname{div} G=0 \text { weakly in } \Omega, \quad \nabla u(\boldsymbol{x})=\int_{\mathbb{R}^{2}} \lambda \mathrm{d} \nu_{x}(\lambda, \rho) .
\end{array}\right.
$$

At this point, the direct computation of $C Q W$ is not possible due to the lack of information on the class of div-curl Young measures. The method is therefore as follows. In a first step, we perform the minimization by retaining just the relevant property expressed in the commutation (3.2), so that we regard feasible measures $\nu$ as Young measures which satisfy this commutation property, but are not necessarily a div-curl Young measure. This provides a lower-bound of $C Q W$. Then, we study whether the optimal measure obtained in this class satisfies the sufficient condition provided by Lemma 3.2 for a Young measure to be a div-curl Young measure.

\subsection{Computation of a lower bound of $C Q W$}

To proceed further with the analysis of this relaxed formulation, we regard $\boldsymbol{x} \in \Omega$ as a parameter and put $\nu_{x}=\nu, G(\boldsymbol{x})=\rho, \nabla u=\lambda$, and $s(\boldsymbol{x})=s$.

Let us take $\nu$ a Young measure which is supported in the set $\Lambda=\Lambda_{\alpha} \cup \Lambda_{\beta}$ where $\Lambda_{\gamma}=\left\{(p, q) \in \mathbb{R}^{2} \times \mathbb{R}^{2}\right.$; $p=\gamma q\}$, a linear manifold in $\mathbb{R}^{2} \times \mathbb{R}^{2}$. We can decompose $\nu=s \nu_{\alpha}+(1-s) \nu_{\beta}$ where $\nu_{\gamma}$ is a probability measure (most likely not a div-curl Young measure itself) supported in $\Lambda_{\gamma}$.

Concerning the first moment of $\nu$, we may write

$$
(\lambda, \rho)=\int_{\Lambda}(p, q) \mathrm{d} \nu(p, q)=s \int_{\mathbb{R}^{2}}(p, \alpha p) \mathrm{d} \nu_{\alpha}^{(1)}(p)+(1-s) \int_{\mathbb{R}^{2}}(p, \beta p) \mathrm{d} \nu_{\beta}^{(1)}(p)
$$


where $\nu_{\gamma}^{(1)}$ is the projection of $\nu_{\gamma}$ onto the first copy of $\mathbb{R}^{2}$ of the product $\mathbb{R}^{2} \times \mathbb{R}^{2}$. By introducing

$$
\lambda_{\gamma}=\int_{\mathbb{R}^{2}} p \mathrm{~d} \nu_{\gamma}^{(1)}(p)
$$

we have $\lambda=s \lambda_{\alpha}+(1-s) \lambda_{\beta}, \rho=s \alpha \lambda_{\alpha}+(1-s) \beta \lambda_{\beta}$, and then

$$
\lambda_{\alpha}=\frac{1}{s(\beta-\alpha)}(\beta \lambda-\rho), \quad \lambda_{\beta}=\frac{1}{(1-s)(\beta-\alpha)}(\rho-\alpha \lambda) .
$$

Moreover, the commutation with the inner product yields the relation

$$
\lambda^{T} \rho=\int_{\Lambda} p^{T} q \mathrm{~d} \nu(p, q)=\alpha s \int_{\mathbb{R}^{2}} p^{T} p \mathrm{~d} \nu_{\alpha}^{(1)}(p)+\beta(1-s) \int_{\mathbb{R}^{2}} p^{T} p \mathrm{~d} \nu_{\beta}^{(1)}(p) .
$$

We now use the commutation (3.2). Introduce

$$
X_{\gamma}=\int_{\mathbb{R}^{2}} p p^{T} \mathrm{~d} \nu_{\gamma}^{(1)}(p), \quad \gamma=\alpha, \beta
$$

a convex combination of symmetric rank-one matrices. It is well-known that

$$
X_{\gamma} \geq \lambda_{\gamma} \lambda_{\gamma}^{T}, \quad \gamma=\alpha, \beta
$$

in the usual sense of symmetric matrices, i.e. that $X_{\gamma}-\lambda_{\gamma} \lambda_{\gamma}^{T}$ is semi-definite positive. The relation (3.2) becomes

$$
\lambda^{T} \rho=\lambda \cdot \rho=\alpha s \operatorname{Tr}\left(X_{\alpha}\right)+\beta(1-s) \operatorname{Tr}\left(X_{\beta}\right)
$$

where $\operatorname{Tr}$ designates the trace operator for square matrices. Similarly, the cost may be written in term of the variable $X_{\gamma}$ as follows:

$$
s \alpha A_{\psi}: X_{\alpha}+(1-s) \beta A_{\psi}: X_{\beta}=\operatorname{s\alpha Tr}\left(A_{\psi} X_{\alpha}\right)+(1-s) \beta \operatorname{Tr}\left(A_{\psi} X_{\beta}\right)
$$

from the relation $A_{\psi}: X_{\gamma}=\operatorname{Tr}\left(A_{\psi} X_{\gamma}\right), \gamma=\alpha, \beta$. Consequently, in seeking a lower bound of the constrained quasi-convex envelope, we are led to consider the mathematical programming problem

$$
\min _{X_{\alpha}, X_{\beta}} C\left(X_{\alpha}, X_{\beta}\right)=\alpha s \operatorname{Tr}\left(A_{\psi} X_{\alpha}\right)+\beta(1-s) \operatorname{Tr}\left(A_{\psi} X_{\beta}\right)
$$

subject to the constraints

$$
\lambda^{T} \rho=\lambda \cdot \rho=\alpha s \operatorname{Tr}\left(X_{\alpha}\right)+\beta(1-s) \operatorname{Tr}\left(X_{\beta}\right), \quad X_{\gamma} \geq \lambda_{\gamma} \lambda_{\gamma}^{T}
$$

We first realize that the set of vectors $(\lambda, \rho)$ for which the constraints yield a non-empty set, are precisely those for which

$$
\alpha s \operatorname{Tr}\left(\lambda_{\alpha} \lambda_{\alpha}^{T}\right)+\beta(1-s) \operatorname{Tr}\left(\lambda_{\beta} \lambda_{\beta}^{T}\right) \leq \lambda \cdot \rho
$$

i.e. if

$$
B_{1} \equiv \lambda \cdot \rho-\alpha s\left|\lambda_{\alpha}\right|^{2}-\beta(1-s)\left|\lambda_{\beta}\right|^{2} \geq 0
$$

using that $\operatorname{Tr}\left(\lambda \rho^{T}\right)=\lambda \cdot \rho$. This inequality, related to the state equation in (1.2), is the usual one obtained, for instance, in the so-called compliance problem (see [32]). Notice that by using (3.19), $B_{1}$ may be factorized as follows: $B_{1}=-s(1-s)\left(\lambda_{\beta}-\lambda_{\alpha}\right) \cdot\left(\beta \lambda_{\beta}-\alpha \lambda_{\alpha}\right)$. We now solve the mathematical programming problem (3.25)-(3.26) in the diagonal and non-diagonal cases, respectively, highlighted in Remark 2.1. 


\subsubsection{Case $A_{\psi}$ diagonal}

We first focus on the diagonal situation $A_{\psi}=\operatorname{diag}\left(\psi_{1,1},-\psi_{1,1}\right)$, for which the cost is simply

$$
C\left(X_{\alpha}, X_{\beta}\right)=\frac{1}{2} \psi_{1,1}\left(\alpha s\left(X_{\alpha, 11}-X_{\alpha, 22}\right)+\beta(1-s)\left(X_{\beta, 11}-X_{\beta, 22}\right)\right)
$$

under the constraints

$$
\left\{\begin{array}{l}
s \alpha\left(X_{\alpha, 11}+X_{\alpha, 22}\right)+(1-s) \beta\left(X_{\beta, 11}+X_{\beta, 22}\right)=\lambda \cdot \rho \\
X_{\gamma, 11}+X_{\gamma, 22} \geq \lambda_{\gamma, 1}^{2}+\lambda_{\gamma, 2}^{2}=\left|\lambda_{\gamma}\right|^{2}, \quad \gamma=\alpha, \beta \\
\left(X_{\gamma, 11}-\lambda_{\gamma, 1}^{2}\right)\left(X_{\gamma, 22}-\lambda_{\gamma, 2}^{2}\right) \geq\left(X_{\gamma, 12}-\lambda_{\gamma, 1} \lambda_{\gamma, 2}\right)^{2} .
\end{array}\right.
$$

Let us first consider the point $\boldsymbol{x}$ of $\Omega$ for which $\psi_{1,1}(\boldsymbol{x}) \geq 0$. Using the first constraint, we write that $s \alpha X_{\alpha, 22}+$ $(1-s) \beta X_{\beta, 22}=\lambda \cdot \rho-s \alpha X_{\alpha, 11}+(1-s) \beta X_{\beta, 11}$, so that the cost simply becomes

$$
C\left(X_{\alpha}, X_{\beta}\right)=\psi_{1,1}\left(s \alpha X_{\alpha, 11}+(1-s) \beta X_{\beta, 11}\right)-\frac{1}{2} \psi_{1,1} \lambda \cdot \rho .
$$

The minimum is then reached for $X_{\gamma, 11}=\max \left(\lambda_{\gamma, 1}^{2},\left|\lambda_{\gamma}\right|^{2}-X_{\gamma, 22}\right)$ since $X_{\gamma, 11} \geq \lambda_{\gamma, 1}^{2}$. By using that $X_{\gamma, 22} \geq \lambda_{\gamma, 2}^{2}$, the maximum is $\lambda_{\gamma, 1}^{2}$. Consequently,

$$
X_{\gamma, 11}=\lambda_{\gamma, 1}^{2}, \quad X_{\gamma, 22} \geq \lambda_{\gamma, 2}^{2}, \quad \gamma=\alpha, \beta
$$

The last constraint then provides the equality

$$
X_{\gamma, 12}=\lambda_{\gamma, 1} \lambda_{\gamma, 2}, \quad \gamma=\alpha, \beta
$$

The cost is then, from (3.19),

$$
C\left(X_{\alpha}, X_{\beta}\right)=\psi_{1,1}\left(\frac{\alpha\left(\beta \lambda_{1}-\rho_{1}\right)^{2}}{s(\beta-\alpha)^{2}}+\frac{\beta\left(\rho_{1}-\alpha \lambda_{1}\right)^{2}}{(1-s)(\beta-\alpha)^{2}}\right)-\frac{1}{2} \psi_{1,1} \lambda \cdot \rho .
$$

Similarly, the study of the case $\psi_{1,1}(\boldsymbol{x}) \leq 0$ leads to

$$
X_{\gamma, 11} \geq \lambda_{\gamma, 1}^{2}, \quad X_{\gamma, 22}=\lambda_{\gamma, 2}^{2}, \quad X_{\gamma, 12}=\lambda_{\gamma, 1} \lambda_{\gamma, 2}, \quad \gamma=\alpha, \beta,
$$

and a cost equal to

$$
C\left(X_{\alpha}, X_{\beta}\right)=-\psi_{1,1}\left(\frac{\alpha\left(\beta \lambda_{2}-\rho_{2}\right)^{2}}{s(\beta-\alpha)^{2}}+\frac{\beta\left(\rho_{2}-\alpha \lambda_{2}\right)^{2}}{(1-s)(\beta-\alpha)^{2}}\right)+\frac{1}{2} \psi_{1,1} \lambda \cdot \rho .
$$

We then obtain the following partial result:

Proposition 3.1 (diagonal case). For any $s \in L^{\infty}(\Omega)$ and $(\lambda, \rho)=(\nabla u, G)$ satisfying (3.12), the function

$$
m(s, \lambda, \rho)=\left\{\begin{array}{lr}
\left|\psi_{1,1}\right|\left(\frac{\alpha\left(\beta \lambda_{1}-\rho_{1}\right)^{2}}{s(\beta-\alpha)^{2}}+\frac{\beta\left(\rho_{1}-\alpha \lambda_{1}\right)^{2}}{(1-s)(\beta-\alpha)^{2}}\right)-\frac{1}{2}\left|\psi_{1,1}\right| \lambda \cdot \rho & \text { if (3.27) } \\
+\infty & \text { else }
\end{array}\right.
$$

is a lower bound for the constrained quasi-convexified $C Q W$ of $W$ :

$$
m(s, \lambda, \rho) \leq C Q W(s, \lambda, \rho) .
$$




\subsubsection{Case $A_{\psi}$ non-diagonal}

We now assume that $A_{\psi}$ is of the form (2.8):

$$
A_{\psi}=\frac{1}{2}\left(\begin{array}{cc}
\psi_{1,1} & 2 \psi_{1,2} \\
0 & -\psi_{1,1}
\end{array}\right) \equiv\left(\begin{array}{cc}
a & 2 b \\
0 & -a
\end{array}\right)
$$

so that the mathematical programming problem is now

$$
\min _{X_{\gamma, 11}, X_{\gamma, 22}, X_{\gamma, 12}} C\left(X_{\alpha}, X_{\beta}\right)
$$

with

$$
C\left(X_{\alpha}, X_{\beta}\right)=\alpha s\left[a\left(X_{\alpha, 11}-X_{\alpha, 22}\right)+2 b X_{\alpha, 12}\right]+\beta(1-s)\left[\left(a\left(X_{\beta, 11}-X_{\beta, 22}\right)+2 b X_{\beta, 12}\right)\right]
$$

and the constraint

$$
\left\{\begin{array}{l}
s \alpha\left(X_{\alpha, 11}+X_{\alpha, 22}\right)+(1-s) \beta\left(X_{\beta, 11}+X_{\beta, 22}\right)=\lambda \cdot \rho, \\
X_{\gamma, 11}+X_{\gamma, 22} \geq \lambda_{\gamma, 1}^{2}+\lambda_{\gamma, 2}^{2}=\left|\lambda_{\gamma}\right|^{2}, \quad \gamma=\alpha, \beta \\
\left(X_{\gamma, 11}-\lambda_{\gamma, 1}^{2}\right)\left(X_{\gamma, 22}-\lambda_{\gamma, 2}^{2}\right) \geq\left(X_{\gamma, 12}-\lambda_{\gamma, 1} \lambda_{\gamma, 2}\right)^{2} .
\end{array}\right.
$$

With the change of variable $Y_{\gamma}=X_{\gamma}-\lambda_{\gamma} \lambda_{\gamma}^{T}$, the cost and the constraints are transformed into

$$
\min _{Y_{\gamma, 11}, Y_{\gamma, 22}, Y_{\gamma, 12}} \alpha s\left(a\left(Y_{\alpha, 11}-Y_{\alpha, 22}\right)+2 b Y_{\alpha, 12}\right)+\beta(1-s)\left(\left(a\left(Y_{\beta, 11}-Y_{\beta, 22}\right)+2 b Y_{\beta, 12}\right)\right)+B_{2}
$$

and

$$
\left\{\begin{array}{l}
s \alpha\left(Y_{\alpha, 11}+Y_{\alpha, 22}\right)+(1-s) \beta\left(Y_{\beta, 11}+Y_{\beta, 22}\right)=B_{1}, \\
Y_{\gamma, 11}+Y_{\gamma, 22} \geq 0, \quad Y_{\gamma, 11} Y_{\gamma, 22} \geq Y_{\gamma, 12}^{2}, \quad \gamma=\alpha, \beta,
\end{array}\right.
$$

where the constants $B_{1}$ and $B_{2}$ are defined by (3.28) and

$$
B_{2}=\alpha s\left(a\left(\lambda_{\alpha, 1}^{2}-\lambda_{\alpha, 2}^{2}\right)+2 b \lambda_{\alpha, 1} \lambda_{\alpha, 2}\right)+\beta(1-s)\left(\left(a\left(\lambda_{\beta, 1}^{2}-\lambda_{\beta, 2}^{2}\right)+2 b \lambda_{\beta, 1} \lambda_{\beta, 2}\right)\right)
$$

respectively. We first realize that the minimum of the linear cost is reached on the boundary of the convex sets

$$
\Gamma_{\gamma}=\left\{\left(Y_{\gamma, 11}, Y_{\gamma, 22}, Y_{\gamma, 12}\right) \in \mathbb{R}^{3}, Y_{\gamma, 11} \geq 0, Y_{\gamma, 22} \geq 0, Y_{\gamma, 11} Y_{\gamma, 22} \geq Y_{\gamma, 12}^{2}\right\}, \quad \gamma=\alpha, \beta
$$

which implies necessarily the equality $Y_{\gamma, 11} Y_{\gamma, 22}=Y_{\gamma, 12}^{2}$. Therefore, we can introduce the new variables $Z_{\gamma} \equiv\left(Z_{\gamma, 11}, Z_{\gamma, 22}\right)^{T}$ so that $Y_{\gamma, 11}=Z_{\gamma, 11}^{2}, Y_{\gamma, 22}=Z_{\gamma, 22}^{2}$ and $\epsilon_{\gamma}= \pm 1$ and then $Z_{\gamma, 11} Z_{\gamma, 22}=\epsilon_{\gamma} Y_{\gamma, 12}$, reducing the problem to

$$
\begin{aligned}
\min _{Z_{\gamma, 11}, Z_{\gamma, 22}, \epsilon_{\gamma}} C\left(Z_{\gamma}, \epsilon_{\gamma}\right)=\alpha s & \left(a\left(Z_{\alpha, 11}^{2}-Z_{\alpha, 22}^{2}\right)+2 b \epsilon_{\alpha} Z_{\alpha, 11} Z_{\alpha, 22}\right) \\
& +\beta(1-s)\left(\left(a\left(Z_{\beta, 11}^{2}-Z_{\beta, 22}^{2}\right)+2 b \epsilon_{\beta} Z_{\beta, 11} Z_{\beta, 22}\right)\right)+B_{2}
\end{aligned}
$$

under the constraint

$$
s \alpha\left(Z_{\alpha, 11}^{2}+Z_{\alpha, 22}^{2}\right)+(1-s) \beta\left(Z_{\beta, 11}^{2}+Z_{\beta, 22}^{2}\right)=B_{1} .
$$

Introducing the Lagrangian $L$ and the multiplier $p$

$$
L\left(Z_{\gamma}, p\right)=C\left(Z_{\gamma}, \epsilon_{\gamma}\right)-p\left(s \alpha\left(Z_{\alpha, 11}^{2}+Z_{\alpha, 22}^{2}\right)+(1-s) \beta\left(Z_{\beta, 11}^{2}+Z_{\beta, 22}^{2}\right)-B_{1}\right),
$$


we arrive at the optimality conditions:

$$
A_{\psi, \epsilon_{\gamma}} Z_{\gamma}=p Z_{\gamma}, \quad A_{\psi, \epsilon_{\gamma}}=\left(\begin{array}{cc}
a & b \epsilon_{\gamma} \\
b \epsilon_{\gamma} & -a
\end{array}\right) .
$$

The trivial solution is $\left(Z_{\gamma, 11}, Z_{\gamma, 22}\right)=(0,0)$ leading to the value of the cost $C\left(Z_{\gamma}, \epsilon_{\gamma}\right)=B_{2}$. The other cases lead to the resolution of a spectral problem: we obtain

$$
p=-\sqrt{a^{2}+b^{2}}, \quad Z_{\gamma}=a_{\gamma}\left(b \epsilon_{\gamma},-\left(a+\sqrt{a^{2}+b^{2}}\right)\right)^{T}
$$

and

$$
p=\sqrt{a^{2}+b^{2}}, \quad Z_{\gamma}=a_{\gamma}\left(b \epsilon_{\gamma},-\left(a-\sqrt{a^{2}+b^{2}}\right)\right)^{T}
$$

for any $a_{\gamma} \in \mathbb{R}^{*}$. Now, writing that $a\left(Z_{\gamma, 11}^{2}-Z_{\gamma, 22}^{2}\right)+2 b \epsilon_{\gamma} Z_{\gamma, 11} Z_{\gamma, 22}=A_{\psi, \epsilon_{\gamma}} Z_{\gamma} \cdot Z_{\gamma}$, we may deduce from (3.50) that

$$
\begin{aligned}
C\left(Z_{\gamma}, \epsilon_{\gamma}\right) & =\alpha s A_{\psi, \epsilon_{\alpha}} Z_{\alpha} \cdot Z_{\alpha}+\beta(1-s) A_{\psi, \epsilon_{\gamma}} Z_{\beta} \cdot Z_{\beta}+B_{2} \\
& =p\left(\alpha s\left|Z_{\alpha}\right|^{2}+\beta(1-s)\left|Z_{\beta}\right|^{2}\right)+B_{2}
\end{aligned}
$$

and then conclude from the constraint (3.48) that the cost given by $(3.47)$ is $C\left(Z_{\gamma}, \epsilon_{\gamma}\right)=p B_{1}+B_{2}$. Therefore, the cost, independent of $\epsilon_{\gamma}$, is obtained for the lowest eigenvalue (independent here of the sign of $a$ ):

$$
\min C\left(Z_{\gamma}, \epsilon_{\gamma}\right)=-\sqrt{a^{2}+b^{2}} B_{1}+B_{2}
$$

for $Z_{\gamma}=a_{\gamma}\left(b \epsilon_{\gamma},-\left(a+\sqrt{a^{2}+b^{2}}\right)\right)^{T}$. The constraint (3.48) then gives the relation

$$
\left(a_{\alpha}^{2} s \alpha+a_{\beta}^{2}(1-s) \beta\right)\left(b^{2}+\left(a+\sqrt{a^{2}+b^{2}}\right)^{2}\right)=B_{1}
$$

We then observe that the cost for this non trivial solution is lower (except in the case $B_{1}=0$, i.e. the equality in (3.27)). It is also important for the search of laminates (see Sect. 3.4) to remark that the value of the cost is unchanged if $Z_{\alpha}=0$ or $Z_{\beta}=0$. Precisely, (3.53) remains true. Finally, we check that for $b=0$, we recover the cost of the diagonal case. Consequently, the partial result is as follows:

Proposition 3.2 (non-diagonal case). For any $s \in L^{\infty}(\Omega)$ and $(\lambda, \rho)=(\nabla u, G)$ satisfying $(3.12)$, the function $m(s, \lambda, \rho)=\left\{\begin{array}{r}\frac{1}{2}\left[-\sqrt{\psi_{1,1}^{2}+\psi_{1,2}^{2}}\left(\rho \cdot \lambda-\alpha s\left|\lambda_{\alpha}\right|^{2}-\beta(1-s)\left|\lambda_{\beta}\right|^{2}\right)+\psi_{1,1}\left(\alpha s \lambda_{\alpha, 1}^{2}+(1-s) \beta \lambda_{\beta, 1}^{2}\right)\right. \\ +\infty \\ -\psi_{1,1}\left(\alpha s \lambda_{\alpha, 2}^{2}+(1-s) \beta \lambda_{\beta, 2}^{2}\right)+2 \psi_{1,2}\left(\alpha s \lambda_{\alpha, 1} \lambda_{\alpha, 2}+(1-s) \beta \lambda_{\beta, 1} \lambda_{\beta, 2}\right)\end{array}\right]$ else

is a lower bound for the constrained quasi-convex envelope $C Q W$ of $W$ :

$$
m(s, \lambda, \rho) \leq C Q W(s, \lambda, \rho)
$$

$\lambda_{\gamma}=\lambda_{\gamma}(s, \lambda, \rho), \gamma=\alpha, \beta$ are defined by $(3.19)$. 


\section{4. (Div-curl) laminates and relaxed formulation}

We now study whether or not the optimal measure may be recovered by laminates. This would imply that the constrained quasi-convexified is reached. Except for special examples (like the compliance case, i.e. $A_{\psi}=I d$ - which exhibits first and second order laminates [32]), the search of explicit laminates is difficult. In the case studied here, the situation is actually straightforward because the function $m$ is zero out of the support of the function $\boldsymbol{\psi}$. Let us discuss the non-diagonal case.

In the set $\mathcal{D}$, the material $\beta$ is imposed so that the density $s$ is equal to zero. Therefore, the search of laminates is meaningful only in $\Omega / \mathcal{D}$. According to the computation of Section 3.3.2, the optimal second moments are of the form

$$
X_{\gamma}=\lambda_{\gamma} \lambda_{\gamma}^{T}+a_{\gamma}^{2}\left(\begin{array}{cc}
\psi_{1,2}^{2} & -\psi_{1,2}\left(\psi_{1,1}+\sqrt{\psi_{1,1}^{2}+\psi_{1,2}^{2}}\right) \\
-\psi_{1,2}\left(\psi_{1,1}+\sqrt{\psi_{1,1}^{2}+\psi_{1,2}^{2}}\right) & \left(\psi_{1,1}+\sqrt{\psi_{1,1}^{2}+\psi_{1,2}^{2}}\right)^{2}
\end{array}\right)
$$

leading to the cost $-\sqrt{\psi_{1,1}^{2}+\psi_{1,2}^{2}} B_{1}+B_{2}$. But, on $\Omega / \mathcal{D}$, the radial function $\psi$ is zero so that,

$$
X_{\gamma}=\lambda_{\gamma} \lambda_{\gamma}^{T}, \quad x \in \Omega / \mathcal{D}
$$

i.e. in particular

$$
X_{\gamma, i i}=\int_{\mathbb{R}} p_{i}^{2} \mathrm{~d} \nu_{\gamma}^{(1, i)}\left(p_{i}\right)=\left(\int_{\mathbb{R}} p_{i} \mathrm{~d} \nu_{\gamma}^{1, i}\left(p_{i}\right)\right)^{2}=\left(\lambda_{\gamma, i}\right)^{2}, \quad i=1,2
$$

where $\nu_{\gamma}^{(1, i)}$ denotes the projection of $\nu^{(1)}$ onto the $i$ th copy of $\mathbb{R}^{2}$. From the strict convexity of the square function, this implies that $\nu_{\gamma}^{(1, i)}=\delta_{\lambda_{\gamma, i}}$, i.e.

$$
\nu_{\alpha}^{(1, i)}=\delta_{\frac{\beta \lambda_{i}-\rho_{i}}{s(\beta-\alpha)}}, \quad \nu_{\beta}^{(1, i)}=\delta_{\frac{\rho_{i}-\alpha \lambda_{i}}{(1-s)(\beta-\alpha)}} .
$$

Notice that this is compatible with the third equality $X_{\gamma, 12}=\lambda_{\gamma, 1} \lambda_{\gamma, 2}^{T}$. This also implies (see for instance (3.55)) the equality in (3.27), i.e. that

$$
B_{1}=\lambda \cdot \rho-\alpha s\left|\lambda_{\alpha}\right|^{2}-\beta(1-s)\left|\lambda_{\beta}\right|^{2}=0
$$

Consequently, the optimal value $m(s, \lambda, \rho)$ may be recovered by the following measure

$$
\nu=s \delta_{\left(\alpha \lambda_{\alpha}, \lambda_{\alpha}\right)}+(1-s) \delta_{\left(\beta \lambda_{\beta}, \lambda_{\beta}\right)}
$$

which is a first order (div-curl) laminate since the sufficient condition $\left(\beta \lambda_{\beta}-\alpha \lambda_{\alpha}\right) \cdot\left(\lambda_{\beta}-\lambda_{\alpha}\right)=0$ of Lemma 3.2 is precisely equivalent to $B_{1}=0$. We therefore may establish the following relaxation:

Theorem 3.1. The variational problem

$$
(R P): \quad \min _{s, u, G} \int_{\Omega} m(s, \nabla u, G) \mathrm{d} x
$$

subject to

$$
\left\{\begin{array}{l}
s \in L^{\infty}(\Omega,[0,1]), s=0 \text { in } \mathcal{D} \cup \partial \Omega, \int_{\Omega} s(\boldsymbol{x}) \mathrm{d} x=L|\Omega|, \\
u \in H^{1}(\Omega), \quad u=u_{0} \text { on } \Gamma_{0}, \quad \beta \nabla u \cdot \boldsymbol{\nu}=g \text { on } \Gamma_{g}, \\
G \in\left(L^{2}(\Omega)\right)^{2}, \quad \operatorname{div} G=0 \text { weakly in } \Omega, \quad G=\beta \nabla u \text { in } \mathcal{D}
\end{array}\right.
$$

where $m$ is defined by (3.56) is a relaxation of $(V P)$ in the sense that the minimum of $(R P)$ exists and equals the infimum of $(V P)$. Moreover, the underlying Young measure associated with $(R P)$ can be found in the form 
of a first order laminate whose direction of lamination are given explicitly in terms of the optimal solution $(u, G)$ : the normal is orthogonal to $\lambda_{\beta}-\lambda_{\alpha}$.

The above formulation may be simplified by taking into account that $B_{1}=0$. Precisely, we use (3.19) to express $B_{1}=-s(1-s)\left(\beta \lambda_{\beta}-\alpha \lambda_{\alpha}\right)\left(\lambda_{\beta}-\lambda_{\alpha}\right)=0$ as follows

$$
\left(\rho-\lambda^{-}(s) \lambda\right) \cdot\left(\rho-\lambda^{+}(s) \lambda\right)=0
$$

in terms of the harmonic and arithmetic mean of $\alpha, \beta$ defined by:

$$
\lambda^{-}(s)=\frac{\alpha \beta}{\alpha(1-s)+\beta s}, \quad \lambda^{+}(s)=\alpha s+\beta(1-s) .
$$

Therefore, Theorem 3.1 is equivalent to

Theorem 3.2. The variational problem

$$
(\overline{R P}): \quad \min _{s, u, G} \int_{\Omega} F(s, \nabla u, G) \mathrm{d} x
$$

subject to

where $F$, deduced from $m$, is defined

$$
\left\{\begin{array}{l}
s \in L^{\infty}(\Omega,[0,1]), s=0 \text { in } \mathcal{D} \cup \partial \Omega, \int_{\Omega} s(\boldsymbol{x}) \mathrm{d} x=L|\Omega|, \\
u \in H^{1}(\Omega), \quad u=u_{0} \text { on } \Gamma_{0}, \quad \beta \nabla u \cdot \boldsymbol{\nu}=g \text { on } \Gamma_{g}, \\
G \in\left(L^{2}(\Omega)\right)^{2}, \quad \operatorname{div} G=0 \text { weakly in } \Omega, \quad G=\beta \nabla u \text { in } \mathcal{D}, \\
\left(G-\lambda^{-}(s) \nabla u\right) \cdot\left(G-\lambda^{+}(s) \nabla u\right)=0 \text { in } L^{2}(\Omega),
\end{array}\right.
$$

$F(s, \lambda, \rho)=\frac{1}{2}\left[\psi_{1,1}\left(\alpha s \lambda_{\alpha, 1}^{2}+(1-s) \beta \lambda_{\beta, 1}^{2}\right)-\psi_{1,1}\left(\alpha s \lambda_{\alpha, 2}^{2}+(1-s) \beta \lambda_{\beta, 2}^{2}\right)+2 \psi_{1,2}\left(\alpha s \lambda_{\alpha, 1} \lambda_{\alpha, 2}+(1-s) \beta \lambda_{\beta, 1} \lambda_{\beta, 2}\right)\right]$

is a relaxation of $(V P)$ in the sense that the minimum of $(\overline{R P})$ exists and equals the minimum of $(V P)$.

\subsection{A final transformation}

The above analysis provides an explicit relaxation $(\overline{R P})$, in terms of a minimum of a new functional over a convex set. This formulation is not standard since the state equation (1.2) under a usual divergence form has disappeared. This state equation is incorporated in the constraints (3.69). This fact explains why a direct numerical approximation (a priori, the nonlinear problem $(\overline{R P})$ cannot be solved analytically) of this problem is difficult. In order to overcome somehow this point, we stress that the relation (3.66) is equivalent to

$$
\left|\rho-\frac{\lambda^{+}(s)+\lambda^{-}(s)}{2} \lambda\right|^{2}=\left(\frac{\lambda^{+}(s)-\lambda^{-}(s)}{2}\right)^{2}|\lambda|^{2} .
$$

Therefore, by introducing the additional variable $t(\boldsymbol{x}) \in \mathbb{R}^{2}$ such that $|t|=1$, we may write $\rho=G(\boldsymbol{x})$ for all $\boldsymbol{x} \in \Omega$ under the form (we use that $\lambda^{-}(s) \leq \lambda^{+}(s)$ for all $s \in(0,1)$ )

$$
\rho=\frac{\lambda^{+}(s)+\lambda^{-}(s)}{2} \lambda+\frac{\lambda^{+}(s)-\lambda^{-}(s)}{2}|\lambda| t \equiv \phi(s, t, \lambda) .
$$

We have

$$
\frac{\lambda^{+}(s)+\lambda^{-}(s)}{2}=\frac{2 \alpha \beta+s(1-s)(\beta-\alpha)^{2}}{2(\alpha(1-s)+\beta s)} \equiv A(s)
$$


and

$$
\frac{\lambda^{+}(s)-\lambda^{-}(s)}{2}=\frac{s(1-s)(\beta-\alpha)^{2}}{2(\alpha(1-s)+\beta s)} \equiv B(s) .
$$

The relation $\operatorname{div} G=0$ then permits to recover $u$ as the solution of a nonlinear equation under a divergence form (having in mind that $\lambda=\nabla u$ ):

$$
\begin{cases}\operatorname{div}(A(s) \nabla u+B(s)|\nabla u| t)=0, & \text { in } \Omega, \\ u=u_{0}, & \text { on } \Gamma_{0}, \\ \beta \nabla u \cdot \boldsymbol{\nu}=g, & \text { on } \Gamma_{g} .\end{cases}
$$

The study - out of the scope of this work - of this apparently non standard elliptic equation would be very interesting. We assume here that (3.75) is well-posed. Note that the nonlinear part vanishes where $s$ takes the values in $\{0,1\}$ since $B(0)=B(1)=0$. In such a case, (3.75) is nothing but (1.2). The relaxed problem is then equivalent to the following one, easier to solve numerically, although nonlinear in $u$ and non standard:

Theorem 3.3. Let $F$ and $\phi$ be defined respectively by (3.70) and (3.72). The following formulation

$$
(\underline{R P}): \quad \min _{s, t} I(s, t)=\int_{\Omega} F(s, \nabla u, \phi(s, t, \nabla u)) \mathrm{d} x
$$

subject to the constraints

$$
\left\{\begin{array}{l}
s \in L^{\infty}(\Omega,[0,1]), s=0 \text { in } \mathcal{D} \cup \partial \Omega, \int_{\Omega} s(\boldsymbol{x}) \mathrm{d} x=L|\Omega|, \\
t \in L^{\infty}\left(\Omega, \mathbb{R}^{2}\right), \quad|t|=1, \\
u \in H^{1}(\Omega), \quad u=u_{0} \text { on } \Gamma_{0}, \quad \beta \nabla u \cdot \nu=g \text { on } \Gamma_{g}, \\
\operatorname{div} \phi(s, t, \nabla u)=0 \text { weakly in } \Omega
\end{array}\right.
$$

is equivalent to the relaxation $(R P)$. In particular, $(\underline{R P})$ is a full well-posed relaxation of $(V P)$.

Remark 3.1. Using the fact that the density $s$ is identically zero on $\{\boldsymbol{x} \in \Omega, \psi(\boldsymbol{x}) \neq 0\}$, one may simplify a bit more the integrand $F$. Using (3.19) and (3.72), we explicitly write $\lambda_{\gamma}$ in terms of $\lambda, s$ and $t$ :

$$
\begin{aligned}
& \lambda_{\alpha}=\frac{2 \beta-(1-s)(\beta-\alpha)}{2(\alpha+s(\beta-\alpha))} \lambda-\frac{(1-s)(\beta-\alpha)}{2(\alpha+s(\beta-\alpha))}|\lambda| t, \\
& \lambda_{\beta}=\frac{s(\beta-\alpha)+2 \alpha}{2(\alpha+s(\beta-\alpha))} \lambda+\frac{s(\beta-\alpha)}{2(\alpha+s(\beta-\alpha))}|\lambda| t,
\end{aligned}
$$

and compute that

$$
F(0, \lambda, \phi(0, t, \lambda))=\frac{1}{2} \beta \psi_{1,1}\left(\lambda_{1}^{2}-\lambda_{2}^{2}\right)+\beta \psi_{1,2} \lambda_{1} \lambda_{2} .
$$

Since $s=0$ in $\{\boldsymbol{x} \in \Omega, \psi(\boldsymbol{x}) \neq 0\}$, we deduce that (taking $\lambda=\nabla u$ )

$$
F(s, \nabla u, \phi(s, t, \nabla u))=\frac{1}{2} \beta \psi_{1,1}\left(u_{, 1}^{2}-u_{, 2}^{2}\right)+\beta \psi_{1,2} u, 1 u_{, 2}
$$

which is nothing but the integrand of $\mathcal{T}_{\boldsymbol{\psi}}$. Therefore, the relaxation of $(P)$ is simply apparent through the nonlinear state equation (3.75). 


\section{NUMERICAL STUDY}

We illustrate in this section our theoretical results by some numerical developments. We first provide some details about the numerical resolution of the relaxed formulation $(\underline{R P})$, and then show some numerical examples.

\subsection{Numerical resolution of the relaxed problem $(\underline{R P})$}

Since $u$ is completely determined by $s$ and $t$, the minimization of the cost is over $s$ and $t$ using a first order gradient method. We compute explicitly the first variation of $I$ with respect to $s$ and $t$ in the direction $\delta s$ and $\delta t$, defined (formally) as:

$$
\frac{\partial I(s, t)}{\partial s} \cdot \delta s=\lim _{\eta \rightarrow 0} \frac{I(s+\eta \delta s, t)-I(s, t)}{\eta}, \quad \eta \in \mathbb{R}^{*}
$$

and

$$
\frac{\partial I(s, t)}{\partial t} \cdot \delta t=\lim _{\eta \rightarrow 0} \frac{I(s, t+\eta \delta t)-I(s, t)}{\eta}, \quad \eta \in \mathbb{R}^{*} .
$$

Theorem 4.1. The first variation of $I$ with respect to $s$ and $t$ in the direction $\delta s$ and $\delta t$ exist and are given respectively by

$$
\frac{\mathrm{d} \mathcal{I}(s, t, u, p)}{\mathrm{d} s} \cdot \delta s=\int_{\Omega} F_{, s}(s, \nabla u, \phi(s, t, \nabla u)) \cdot \delta s \mathrm{~d} x+\int_{\Omega}\left(A_{, s}(s) \nabla u \cdot \nabla p+B_{, s}(s)|\nabla u| t \cdot \nabla p\right) \cdot \delta s \mathrm{~d} x
$$

and

$$
\frac{\mathrm{d} \mathcal{I}(s, t, u, p)}{\mathrm{d} t} \cdot \delta t=\int_{\Omega} F_{, t}(s, \nabla u, \phi(s, t, \nabla u)) \cdot \delta t \mathrm{~d} x+\int_{\Omega} B(s)|\nabla u| \delta t \cdot \nabla p \mathrm{~d} x
$$

where $p \in H_{\Gamma_{0}}^{1}(\Omega)=\left\{v \in H^{1}(\Omega), v=0\right.$ on $\left.\Gamma_{0}\right\}$ solves the adjoint problem

$$
\int_{\Omega} F_{, u}(s, \nabla u, \phi(s, t, \nabla u)) \cdot v \mathrm{~d} x+\int_{\Omega}\left(A(s) \nabla v \cdot \nabla p+B(s) \frac{\nabla u \cdot \nabla v}{|\nabla u|} t \cdot \nabla p\right) \mathrm{d} x=0,
$$

for all $v$ in $H_{\Gamma_{0}}^{1}(\Omega)$. $A_{, s}$ and $B, s$ denote the partial derivative of $A$ and $B$ with respect to $s$ and $F_{, t}$ the partial derivative of $F$ with respect to $t$.

Notice that the adjoint formulation (4.5) is linear in contrast with the formulation associated with $u$, which reads as follows,

$$
\int_{\Omega}(A(s) \nabla u \cdot \nabla v+B(s)|\nabla u| t \cdot \nabla v) \mathrm{d} x=\int_{\Gamma_{g}} g v \mathrm{~d} \sigma, \quad \forall v \in H_{\Gamma_{0}}^{1}(\Omega)
$$

using that $s=0$ on $\partial \Omega$ and that $A(0)=\beta, B(0)=0$.

Proof of Theorem 4.1. The proof is standard. We introduce the Lagrangian

$$
\mathcal{L}(s, t, u, p)=\int_{\Omega} F(s, \nabla u, \phi(s, t, \nabla u)) \mathrm{d} x+\int_{\Omega}(A(s) \nabla u \cdot \nabla p+B(s)|\nabla u| t \cdot \nabla p) \mathrm{d} x-\int_{\Gamma_{g}} g p \mathrm{~d} \sigma
$$

and write that

Formally, we have

$$
\frac{\mathrm{d} \mathcal{L}(s, t, u, p)}{\mathrm{d}(s, t)} \cdot(\delta s, \delta t)=\frac{\mathrm{d} \mathcal{L}(s, t, u, p)}{\mathrm{d} s} \cdot \delta s+\frac{\mathrm{d} \mathcal{L}(s, t, u, p)}{\mathrm{d} t} \cdot \delta t .
$$

$$
\frac{\mathrm{d} \mathcal{L}(s, t, u, p)}{\mathrm{d} s} \cdot \delta s=\frac{\partial \mathcal{L}(s, t, u, p)}{\partial s} \cdot \delta s+\left\langle\frac{\partial \mathcal{L}(s, t, u, p)}{\partial u}, \frac{\partial u}{\partial s} \cdot \delta s\right\rangle+\left\langle\frac{\partial \mathcal{L}(s, t, u, p)}{\partial p}, \frac{\partial p}{\partial s} \cdot \delta s\right\rangle .
$$


As usual, since $\mathcal{L}$ is linear in $p$ and since $u$ is the solution of (4.6), the third term is equal to zero. The solution $p$ is then determined in order that the second term be also equal to zero. We write (for simplicity, we write $\delta u$ instead of $(\partial u / \partial s) \cdot \delta s)$

$$
\left\langle\frac{\partial \mathcal{L}(s, t, u, p)}{\partial u}, \delta u\right\rangle=\int_{\Omega} F_{, u}(s, \nabla u, \phi(s, t, \nabla u)) \cdot \delta u \mathrm{~d} x+\int_{\Omega}\left(A(s) \nabla \delta u \cdot \nabla p+B(s) \frac{\nabla u \cdot \nabla \delta u}{|\nabla u|} t \cdot \nabla p\right) \mathrm{d} x
$$

for all $\delta u \in H_{\Gamma_{0}}^{1}(\Omega)$ leading to the linear weak formulation of $p \in H_{\Gamma_{0}}^{1}(\Omega)$. In particular, we check that $\nabla p \cdot \boldsymbol{\nu}=0$ on $\partial \Omega / \Gamma_{0}$. The variation of $\mathcal{L}$ with respect to $s$ is then given by (4.3). Relation (4.4) is obtained in a similar way.

Theorem 4.1 leads to the descent directions

$$
\delta s=-F_{, s}(s, \nabla u, \phi(s, t, \nabla u))-\left(A_{, s}(s) \nabla u \cdot \nabla p+B_{, s}(s)|\nabla u| t \cdot \nabla p\right) \quad \text { in } \Omega
$$

and

$$
\delta t=-\left(F_{, t}(s, \nabla u, \phi(s, t, \nabla u))+B(s)|\nabla u| \nabla p\right) \quad \text { in } \Omega .
$$

Moreover, since $|t|=1$, we introduce the variable $\theta$ and write $t=(\cos (\theta), \sin (\theta))$, in which case (4.12) is replaced by

$$
\delta \theta=-\sin (\theta)(\delta t)_{1}+\cos (\theta)(\delta t)_{2} \quad \text { in } \Omega .
$$

The volume constraint on $s$ is taken into account through a classical and efficient way by introducing an explicit Lagrange multiplier (we refer to [21]). The algorithm for the variable $s$ is therefore

$$
\left\{\begin{array}{l}
s^{(0)} \in L^{\infty}(\Omega,[0,1]), \\
s^{(k+1)}=s^{(k)}+\epsilon f_{s}\left(s^{k}\right) \delta s^{(k)}, \quad f_{s}\left(s^{(k)}\right)=s^{k}\left(1-s^{(k)}\right), \quad k \geq 0
\end{array}\right.
$$

where the positive value $f_{s}\left(s^{(k)}\right)$ is introduced in order to enforce $s^{(k+1)}$ to be in $[0,1]$ and $\epsilon$ a positive real small enough. The descent algorithm for the field $\theta$ is

$$
\theta^{(0)} \in L^{\infty}(\Omega, \mathbb{R}), \quad \theta^{(k+1)}=\theta^{(k)}+\epsilon \delta \theta^{(k)}, \quad k \geq 0 .
$$

At each step $k$ of these two algorithms, the solution $u$ of the nonlinear system (3.75) is solved from (4.6) by using the Newton method:

$$
\left\{\begin{array}{l}
u^{0} \in H^{1}(\Omega), u^{0}=u_{0} \text { on } \Gamma_{0}, \\
\int_{\Omega}\left(A\left(s^{(k)}\right) \nabla u^{n+1} \cdot \nabla v+B\left(s^{(k)}\right) \frac{\nabla u^{n+1} \cdot \nabla u^{n}}{\left|\nabla u^{n}\right|} t^{(k)} \cdot \nabla v\right) \mathrm{d} x=\int_{\Gamma_{g}} g v \mathrm{~d} \sigma, \forall n>0, \forall v \in H_{\Gamma_{0}}^{1}(\Omega) .
\end{array}\right.
$$

This formulation and the linear equation (4.5) for $p$ are solved using continuous finite elements of order one approximating the space $H_{\Gamma_{0}}^{1}(\Omega)$ by the following finite dimensional space:

$$
H_{\Gamma_{0}, h}^{1}(\Omega)=\left\{v_{h}, v_{h} \in C^{0}(\bar{\Omega}),\left.v_{h}\right|_{Q} \in Q_{1}(Q), \forall Q \in Q_{h}, v_{h}=0 \text { on } \Gamma_{0}\right\}
$$

where $Q_{1}(Q)$ denotes the space of polynomial functions of degree $\leq 1$ on $Q$, the notation $\left(Q_{h}\right)_{h>0}$ stands for a regular family of quadrangulations characterized by the space step $h$ such that $\bar{\Omega}=\cup_{Q \in Q_{h}} Q$. We highlight that the corresponding stiffness matrix is identical for (4.5) and (4.16). The lips of the crack $\gamma$, assumed rectilinear, are composed of edges of elements in $Q_{h}$. Besides, as proved in [12] (Thm. 4.2, p. 96), (4.17) implies the a priori estimate $\left|\mathcal{T}_{\psi}-\mathcal{T}_{\psi, h}\right|=O\left(h^{1-\eta}\right), \forall \eta>0$ if $\mathcal{T}_{\psi, h}$ designates the numerical approximation of the energy release rate $\mathcal{T}_{\psi}$. 


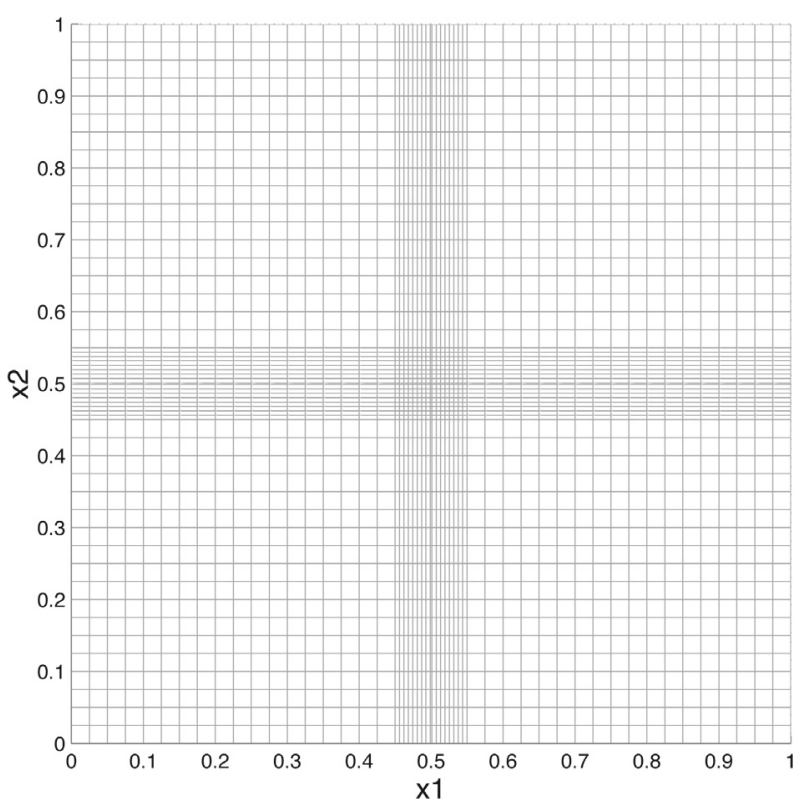

FIGURE 4. Example of quadrangulation of the unit square with a refinement on the support of the radial function $\psi_{1}(52 \times 52$ finite elements $)$ around the point $\boldsymbol{F}=(1 / 2,1 / 2)$.

\subsection{Numerical experiments}

For simplicity, we consider in the sequel the domain $\Omega=(0,1)^{2}$ with the crack $\gamma=[0.5,1] \times\{a\}(a \in(0,1))$. $\Gamma_{0}$ is divided into two parts $\Gamma_{0,1} \cup \Gamma_{0,2}: \Gamma_{0,1}=\{0\} \times[0,1]=\left\{\boldsymbol{x}=\left(x_{1}, x_{2}\right) \in \mathbb{R}^{2}, x_{1}=0, x_{2} \in[0,1]\right\}$ where $u_{0}=0$ and $\Gamma_{0,2}=\{1\} \times[0.5,0.8]$ where $u_{0}=0.5$. There is not normal load: $\Gamma_{g}=\emptyset$.

The examples that we describe in the sequel correspond to the non-diagonal case (2.8) which imposes the material to be constant around $\boldsymbol{F}, \mathcal{D}=\left\{\boldsymbol{x} \in \Omega,\|\boldsymbol{x}-\boldsymbol{F}\| \leq r_{3}\right\}$ (see Rem. 2.1, item (i)). In order to limit the measure of this set, we take $r_{3}$ small enough with respect to the size of the domain, precisely $r_{3}=0.05$. The radial function $\boldsymbol{\psi}=\left(\psi_{1}, 0\right)$ is then defined by $(2.9)$ with $r_{1}=0.015$ and $r_{2}=0.045<r_{3}$. Therefore, $\psi_{1}$ is constant equal to $\nu_{F, 1}=-1$ on $\left\{\boldsymbol{x} \in \Omega,\|\boldsymbol{x}-\boldsymbol{F}\| \leq r_{1}\right\}$ (we recall that this permits to avoid the singularity of $u$ on $\boldsymbol{F}$ ). These small values limit the measure of $\mathcal{D}$ but enforces a very fine mesh where $\psi_{1}>0$ in order to have an accurate approximation of $\mathcal{T}_{\boldsymbol{\psi}}$. We therefore use a non uniform regular quadrangulation $\left(Q_{h}\right)_{h>0}$ of $\Omega$ with a refinement around the crack point. For $\boldsymbol{F}=(1 / 2,1 / 2)$, an example is given on Figure 4, corresponding to $52 \times 52$ elements (and 2916 degrees of freedom). In practice, $20 \times 20$ elements on $\left\{\boldsymbol{x} \in \Omega,\|\boldsymbol{x}-\boldsymbol{F}\| \leq r_{2}\right\}$ are sufficient to obtain an accurate and invariant (with respect to $r_{1}$ and $r_{2}$ ) approximation of the energy release rate. In the sequel, a mesh composed of $82 \times 82$ quadrangles will be used.

We discuss the optimal distribution of the two materials with respect to the values of $(\alpha, \beta)$ and $a$. Let us first take $(\alpha, \beta)=(1,2)$ and $L=2 / 5$ and $a=1 / 2$ so that $\boldsymbol{F}=(1 / 2,1 / 2)$. The iso-value of the density $s^{\text {opt }}$ obtained after 1000 iterations is depicted in Figure 5 . The algorithm is initialized with $\theta^{(0)}=0$ on $\Omega$ and $s^{(0)}=0$ on $\mathcal{D} \cup \partial \Omega$ and constant elsewhere which does not privilege any location for $\omega$. The constant is determined in order to satisfy the volume constraint. At convergence, the cost is $I\left(s^{\text {opt }}, t^{\text {opt }}\right)=2.87 \times 10^{-2}$. This distribution permits to divide by three the cost from the initial guess. The evolution of the cost with respect to the iteration is given in Figure 6-left. At each iteration, around five iterations of the Newton algorithm permit to solve the nonlinear system (3.75) (the Newton algorithm is stopped as soon as the residual is lower than $\left.10^{-10}\right)$. The corresponding solution is given in Figure 6-right. As expected the soft material is located around the part of the boundary where the displacement is imposed. This has the effect to absorb partially the load and reduce his influence on the crack zone. We also observe that the density is almost a characteristic function, 


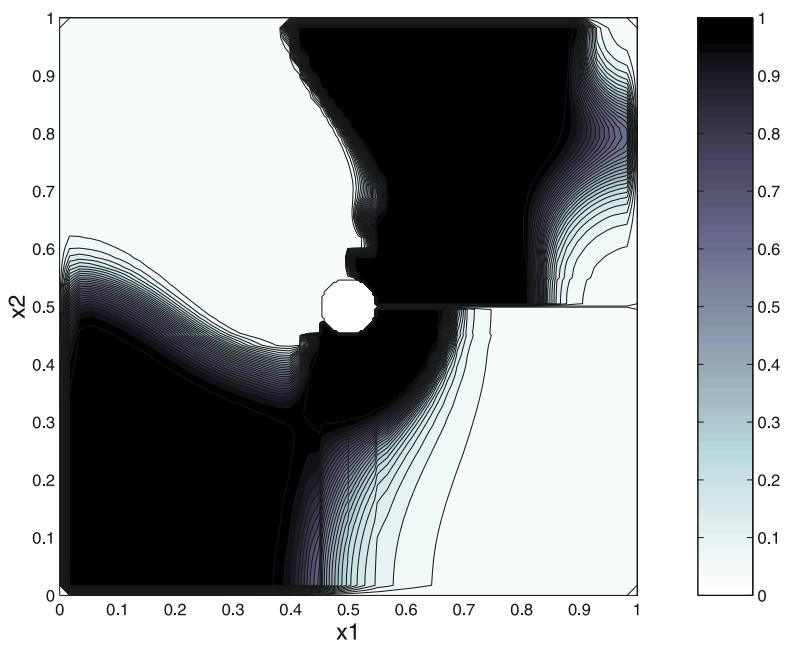

Figure $5 .(\alpha, \beta)=(1,2)-L=2 / 5 ; \boldsymbol{F}=(1 / 2,1 / 2)$ - iso-value of the density $s^{\text {opt }}$ on the crack domain $\Omega$ with $s^{\text {opt }}=0$ on $\partial \Omega$.
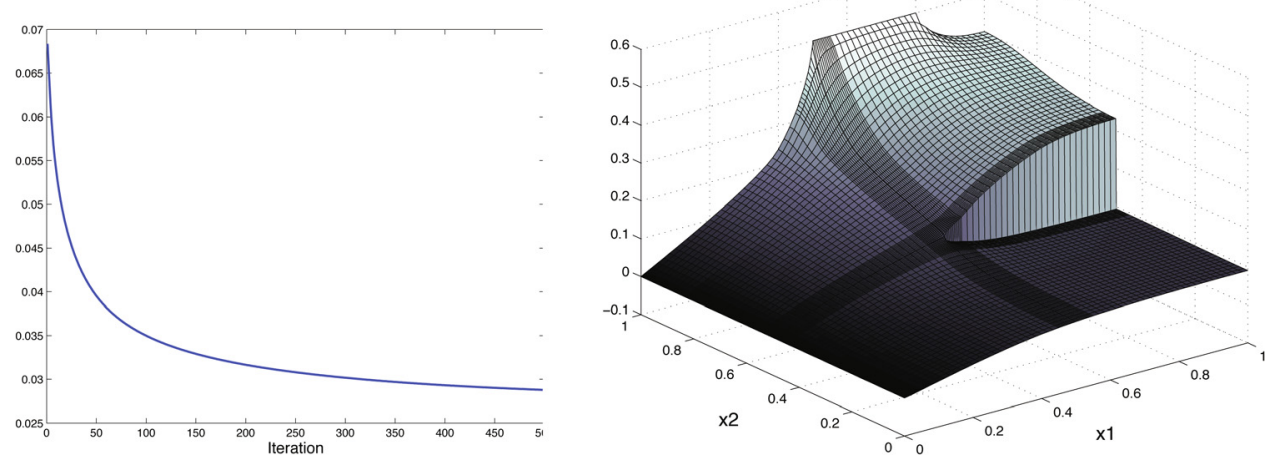

Figure $6 .(\alpha, \beta)=(1,2)-L=2 / 5 ; \boldsymbol{F}=(1 / 2,1 / 2)$ - evolution of the relaxed cost $I\left(s^{(k)}, t^{(k)}\right)$ w.r.t. the iteration (left) and final solution $u$ on $\Omega$ (right).

i.e. a $(0,1)$ function. We check a posteriori that the relation (3.66) holds: we obtain

$$
\left\|\left(\rho-\lambda^{-}\left(s^{\mathrm{opt}}\right) \lambda\right) \cdot\left(\rho-\lambda^{+}\left(s^{\mathrm{opt}}\right) \lambda\right)\right\|_{L^{2}(\Omega)} \approx 1.32 \times 10^{-6}
$$

Moreover, we obtain

$$
\left\|\rho-\lambda^{+}\left(s^{\mathrm{opt}}\right) \lambda\right\|_{L^{2}(\Omega)} \approx 3.13 \times 10^{-4}, \quad\left\|\rho-\lambda^{-}\left(s^{\mathrm{opt}}\right) \lambda\right\|_{L^{2}(\Omega)} \approx 4.21 \times 10^{-3} .
$$

We recall that $\lambda^{-}=\lambda^{+}$for a characteristic function. We also observe that we obtain a similar $\operatorname{cost}\left(I\left(s^{\mathrm{opt}}, t^{\mathrm{opt}}\right) \approx\right.$ $2.84 \times 10^{-2}$ ) when the constraint $s=0$ is relaxed on the boundary, unnecessary here since $\Gamma_{g}$ is empty. The corresponding distribution is given in Figure 7.

As already observed in several different situations ([25] for the heat equation and [20,24,26] for the wave equation), the results are qualitatively different when the gap $\beta-\alpha$ is greater. Figure 8 gives the iso-values 


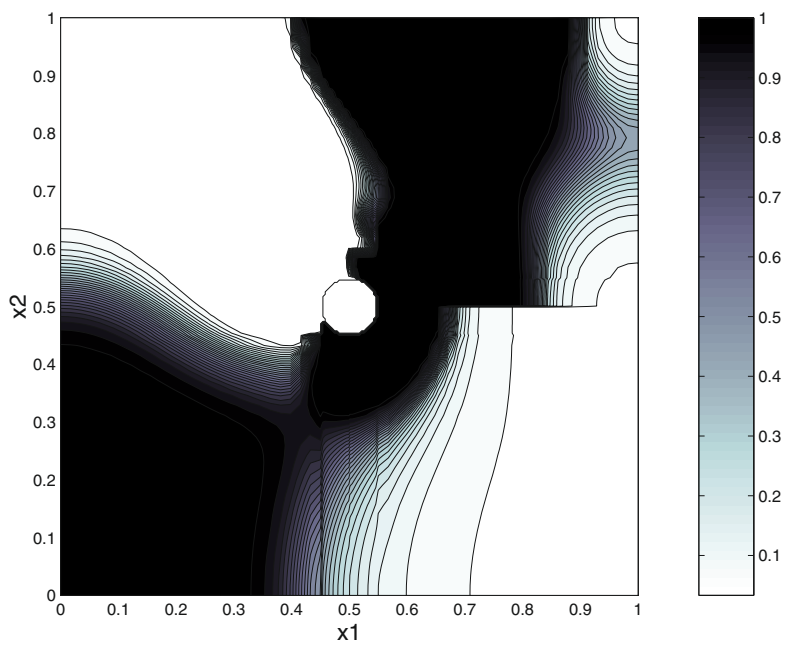

Figure 7. $(\alpha, \beta)=(1,2)-L=2 / 5 ; \boldsymbol{F}=(1 / 2,1 / 2)$ - iso-value of the density $s$ on the crack domain with $s$ free on $\partial \Omega$.

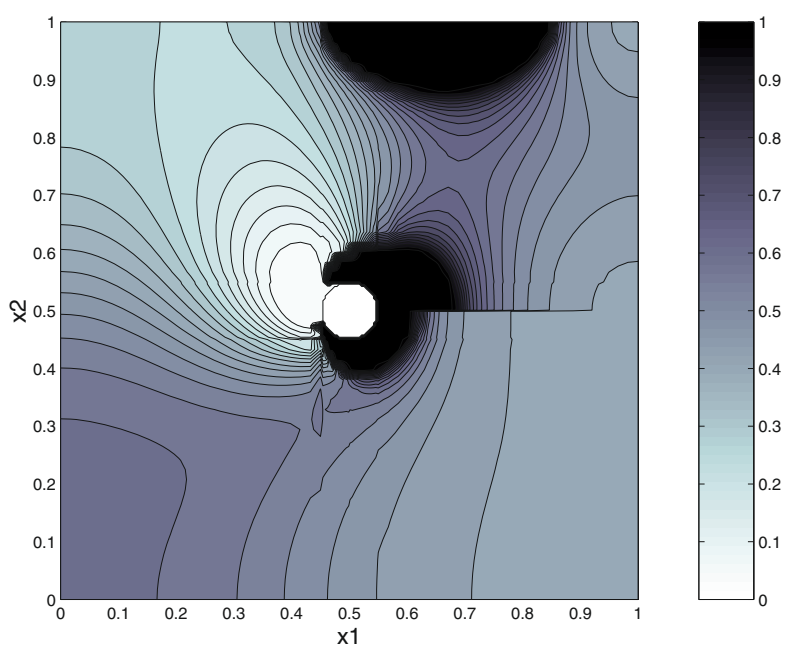

Figure 8. $(\alpha, \beta)=(1,10)-L=2 / 5 ; \boldsymbol{F}=(1 / 2,1 / 2)$ - iso-values of the density $s$ on the crack domain.

of the optimal density for $(\alpha, \beta)=(1,10)$. The cost is $I\left(s^{\mathrm{opt}}, t^{\mathrm{opt}}\right) \approx 1.15 \times 10^{-2}$. This (local) optimal density is no more a $(0,1)$-function. The soft material $\alpha$ is however mainly concentrated around the point $\boldsymbol{F}$ and the part of the boundary $\Gamma_{0,2}$ where $u$ is imposed. This observation justifies the need of relaxation for this problem. In this case, the convergence of the algorithm is slower (Fig. 9-left). Moreover, at each step, the Newton method requires more iterations (around 8): the term $B(s)$ in (3.75) is greater in that case so that the nonlinear term is not negligible with respect to the linear one. Moreover, as expected, the equality (3.66) still holds

$$
\left\|\left(\rho-\lambda^{-}\left(s^{\mathrm{opt}}\right) \lambda\right) \cdot\left(\rho-\lambda^{+}\left(s^{\mathrm{opt}}\right) \lambda\right)\right\|_{L^{2}(\Omega)} \approx 1.32 \times 10^{-5}
$$



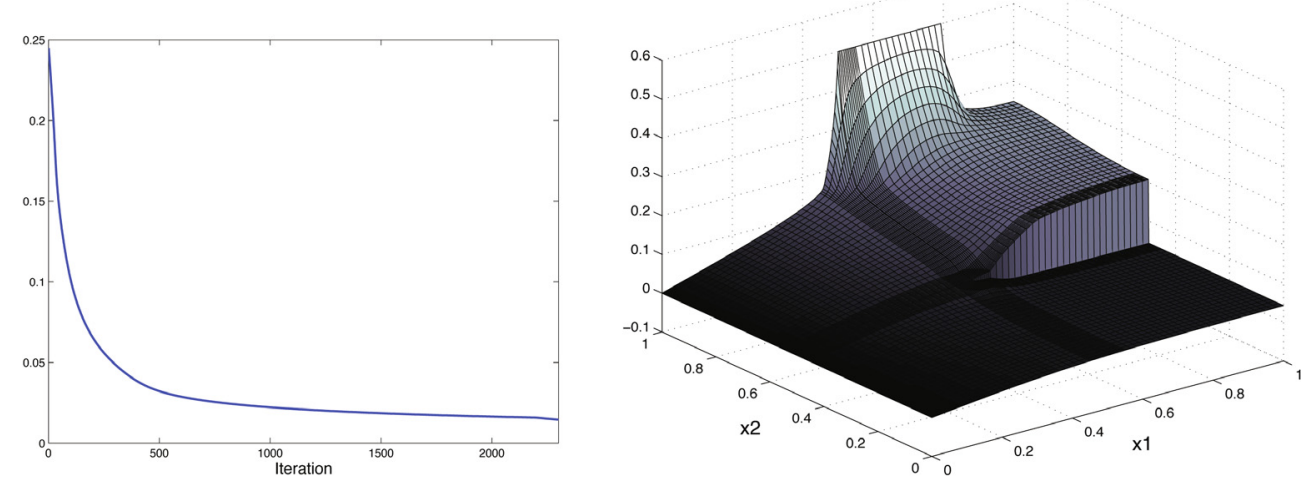

Figure $9 .(\alpha, \beta)=(1,10)-L=2 / 5 ; \boldsymbol{F}=(1 / 2,1 / 2)-\operatorname{relaxed} \operatorname{cost} I\left(s^{(k)}, t^{(k)}\right)$ w.r.t. the iteration (left) and final solution $u$ on $\Omega$ (right).
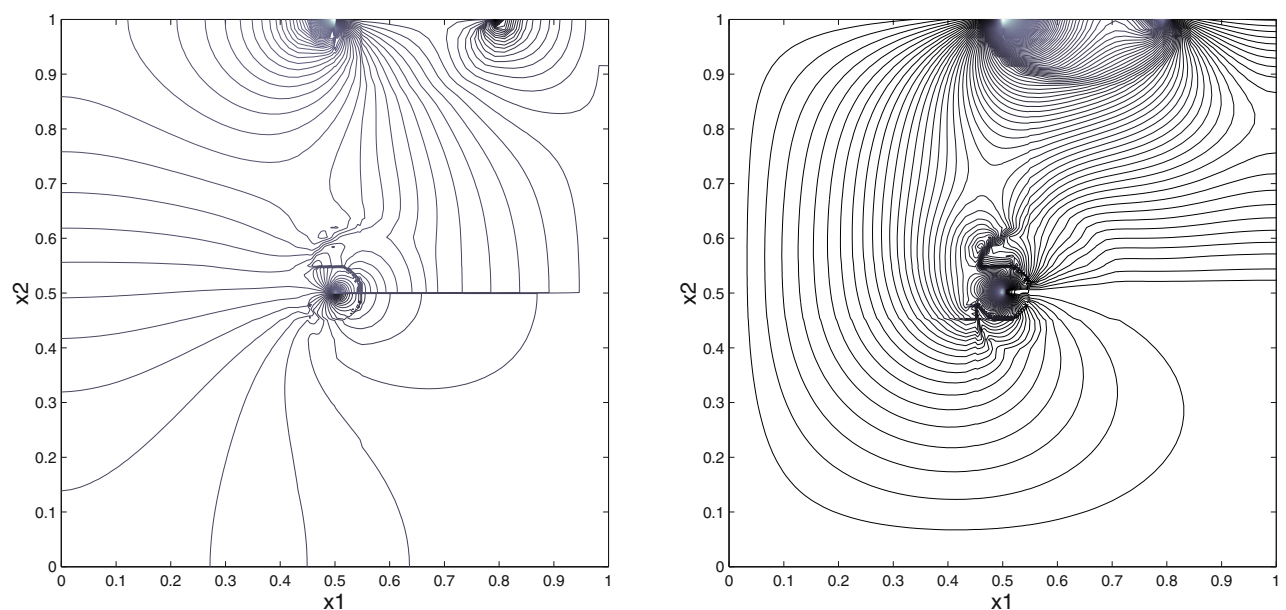

Figure 10. $(\alpha, \beta)=(1,10)-L=2 / 5 ; \boldsymbol{F}=(1 / 2,1 / 2)$ - iso-values of the components of the vector $\lambda_{\beta}-\lambda_{\alpha}$.

but not for the arithmetic nor the harmonic mean:

$$
\left\|\rho-\lambda^{+}\left(s^{\mathrm{opt}}\right) \lambda\right\|_{L^{2}(\Omega)} \approx 8.21 \times 10^{-1}, \quad\left\|\rho-\lambda^{-}\left(s^{\mathrm{opt}}\right) \lambda\right\|_{L^{2}(\Omega)} \approx 4.09 \times 10^{-1} .
$$

If we assume a priori for instance the arithmetic mean, i.e. if we simply replace in $(P) \mathcal{X}_{\omega}$ by $s$ and optimize with respect to $s$, then we obtain a greater cost equal to $4.39 \times 10^{-2}$. The corresponding solution $u$ on the crack domain is depicted in Figure 9-right.

It is then necessary to associate with this optimal composite material a workable shape $\omega$, i.e. to construct a sequence of characteristic function, say $\mathcal{X}_{\omega(k)}$, for which $\mathcal{T}_{\psi}\left(\mathcal{X}_{\omega^{(k)}}\right)$ converges toward $I\left(s^{\text {opt }}, t^{\text {opt }}\right)$. A simple approach, using a local mean argument on $s$, is proposed in [20] to approximate such a sequence. Further, it would be interesting to use the information of the normal of the first-order laminate at each point given by $\lambda_{\beta}-\lambda_{\alpha}$ (we refer to [29] for such analysis in the context of Homogeneisation): iso-values of the vector $\lambda_{\beta}-\lambda_{\alpha}$ are given in Figure 10 . 

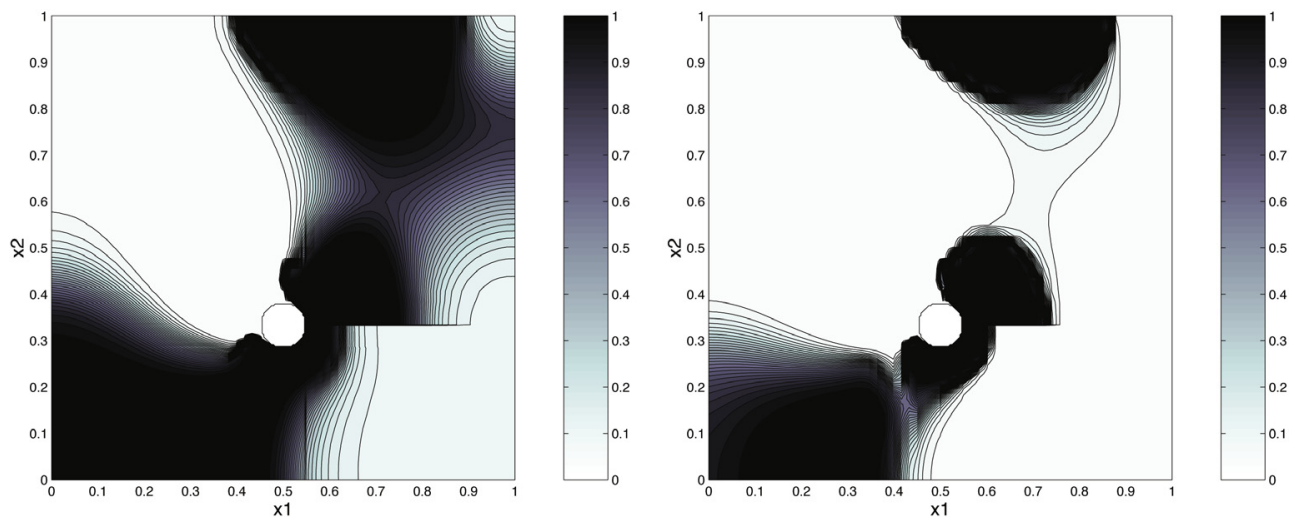

Figure 11. $(\alpha, \beta)=(1,2)-\boldsymbol{F}=(1 / 2,1 / 3)$ - iso-values of the density $s$ for $L=2 / 5$ (left) and $L=1 / 5$ (right).

Finally, similar results are observed for different value of $a$ and $L$. For $a=1 / 3$ corresponding to $\boldsymbol{F}=(1 / 2,1 / 3)$, Figure 11 represents the optimal density obtained for the volume fraction $L=2 / 5$ and $L=1 / 5$. For $L=2 / 5$, we check that the cost $I(s, t) \approx 1.02 \times 10^{-2}$ is lower in that case since the crack point is far away from the load support $\Gamma_{0,2}$.

We also observe that the energy release rate is reduced but not arbitrarily small in spite of the important degree of freedom contained by the shape of $\omega$. Therefore the singularities are not canceled. We suspect that this is due to our mechanical assumption around the point $\boldsymbol{F}$. This also suggests, quite surprisingly, that the optimal distribution around the crack point is not composed only of the harder material.

\section{Concluding Remarks}

To our knowledge, this work is the first one which attempts to minimize the energy release rate, and therefore to control the crack growth, with respect to the conductivity coefficient. This energy release rate presents the originality to be expressed as a scalar product in terms of a non definite positive matrix, in contrast with usual examples such as the energy itself. This apparent difficulty in terms of relaxation is compensated by the mechanical assumption around the crack point. Thus, the variational non-convex approach coupled with Young measures permits to derive an explicit relaxed formulation $(R P)$ of the optimal design problem, involving a nonCarathéodory quasi-convexification. Moreover, the optimal measure is a first-order laminate. Following [33], $(R P)$ is transformed into an equivalent relaxed formulation $(\underline{R P})$ involving an original nonlinear divergenceform system. The numerical experiments suggest that an optimal distribution permits to reduce significantly (with respect to an isotropic one) the cost. However, the optimal cost is not arbitrarily small so that the singularities around the crack tip are not canceled in contrast with $[16,17]$ where the control variable is an additional boundary load. This phenomenon is very likely due to the condition (necessary in our context) which imposes the conductivity to be constant around the crack tip.

This preliminary work would merit to be enriched in several directions. For instance, it is worth to replace the conductivity system by the elastic one (and thus take into account the contact condition on the crack $\gamma$ as it is done in $[16,17]$ using [7]). For the elasticity operator, the full relaxation process is still an open problem. However, the fact that the integrand of the $\operatorname{cost} \mathcal{T}_{\psi}$ is non zero only where the material is uniform may be helpful in the search of div-curl laminates as it is in this work. Moreover, it would be interesting to obtain an optimal distribution independent of the normal load $g \in L^{2}\left(\Gamma_{g}\right)$ and thus consider an inf-sup problem of the type $\inf _{\mathcal{X}_{\omega}} \sup _{\left(g, \Gamma_{g}\right)} \mathcal{T}_{\boldsymbol{\psi}}$. Similarly, in view of the growth of the point $\boldsymbol{F}$, it would be interesting to minimize the rate independently of the length of the crack, assumed straight. Alternatively, following the notion of Dynamic Material introduced recently in [19], one may assume that the conductivity evolves with respect to the crack 
growth along $\Omega$, then solve a sequence of problem $\left(P_{\gamma}\right)_{\gamma}$ and get a non cylindrical optimal distribution of the phases $\alpha$ and $\beta$. Finally, we also plan in the near future to compare the numerical results derived from this relaxation approach with a more direct one based on level set method (we refer to $[4,6,21]$ ). In this direction, we mention the recent work [2] in the framework of damage mechanic.

\section{REFERENCES}

[1] G. Allaire, Shape optimization by the homogenization method, Applied Mathematical Sciences 146. Springer-Verlag, New York (2002).

[2] G. Allaire, F. Jouve and N. Van Goethem, A level set method for the numerical simulation of damage evolution. Internal report 629, CMAP, École polytechnique, France (2007).

[3] H.D. Bui, Mécanique de la rupture fragile. Masson, Paris (1983).

[4] M. Burger, A framework for the construction of level set methods for shape optimization and reconstruction. Interface and Free Boundaries 5 (2003) 301-329.

[5] B. Dacorogna, Direct methods in the calculus of variations, Applied Mathematical Sciences 78. Springer-Verlag, Berlin (1989).

[6] F. De Gournay, G. Allaire and F. Jouve, Shape and topology optimization of the robust compliance via the level set method. ESAIM: COCV 14 (2008) 43-70.

[7] P. Destuynder, Calculation of forward thrust of a crack, taking into account the unilateral contact between the lips of the crack. C. R. Acad. Sci. Paris, Sér. II 296 (1983) 745-748.

[8] P. Destuynder, An approach to crack propagation control in structural dynamics. C. R. Acad. Sci. Paris, Sér. II 306 (1988) 953-956.

[9] P. Destuynder, Remarks on a crack propagation control for stationary loaded structures. C. R. Acad. Sci. Paris, Sér. IIb 308 (1989) 697-701.

[10] P. Destuynder, Computation of an active control in fracture mechanics using finite elements. Eur. J. Mech. A/Solids 9 (1990) 133-141.

[11] P. Destuynder, M. Djaoua and S. Lescure, Quelques remarques sur la mécanique de la rupture élastique. J. Mec. Theor. Appl. 2 (1983) 113-135.

[12] M. Djaoua, Analyse mathématique et numérique de quelques problèmes en mécanique de la rupture. Thèse d'état, Université Paris VI, France (1983).

[13] G.A. Francfort and J.J. Marigo, Revisiting brittle fracture as an energy minimisation problem. J. Mech. Phys. Solids 46 (1998) $1319-1342$

[14] A.A. Griffith, The phenomena of rupture and flow in solids. Phil. Trans. Roy. Soc. London 46 (1920) 163-198.

[15] P. Grisvard, Singularities in boundary value problems, Research in Applied Mathematics. Springer-Verlag, Berlin (1992).

[16] P. Hild, A. Münch and Y. Ousset, On the control of crack growth in elastic media. C. R. Acad. Sci. Paris Sér. Méc. 336 (2008) 422-427.

[17] P. Hild, A. Münch and Y. Ousset, On the active control of crack growth in elastic media. Comput. Methods Appl. Mech. Engrg. 198 (2008) 407-419.

[18] J.-B. Leblond, Mécanique de la rupture fragile et ductile. Hermes Sciences Publications (2003) 1-197.

[19] K.L. Lurie, An introduction to the mathematical theory of dynamic materials, Advances in Mechanics and Mathematics 15. Springer (2007).

[20] F. Maestre, A. Münch and P. Pedregal, A spatio-temporal design problem for a damped wave equation. SIAM J. Appl Math. 68 (2007) 109-132.

[21] A. Münch, Optimal design of the support of the control for the 2-D wave equation: numerical investigations. Int. J. Numer. Anal. Model. 5 (2008) 331-351.

[22] A. Münch and Y. Ousset, Energy release rate for a curvilinear beam. C. R. Acad. Sci. Paris, Sér. IIb 328 (2000) $471-476$.

[23] A. Münch and Y. Ousset, Numerical simulation of delamination growth in curved interfaces. Comput. Methods Appl. Mech. Engrg. 191 (2002) 2045-2067.

[24] A. Münch, P. Pedregal and F. Periago, Optimal design of the damping set for the stabilization of the wave equation. J. Diff. Eq. 231 (2006) 331-358.

[25] A. Münch, P. Pedregal and F. Periago, Relaxation of an optimal design problem for the heat equation. J. Math. Pures Appl. 89 (2008) 225-247.

[26] A. Münch, P. Pedregal and F. Periago, Optimal internal stabilization of the linear system of elasticity. Arch. Rational Mech. Analysis 193 (2009) 171-193.

[27] F. Murat and J. Simon, Études de problèmes d'optimal design. Lect. Notes Comput. Sci. 41 (1976) 54-62.

[28] M.T. Niane, G. Bayili, A. Sène and M. Sy, Is it possible to cancel singularities in a domain with corners and cracks? $C . R$. Acad. Sci. Paris, Sér. I 343 (2006) 115-118. 
[29] O. Pantz and K. Trabelsi, A post-treatment of the homogenization for shape optimization. SIAM J. Control. Optim. 47 (2008) 1380-1398.

[30] P. Pedregal, Parametrized measures and variational principles. Birkhäuser (1997).

[31] P. Pedregal, Vector variational problems and applications to optimal design. ESAIM: COCV 11 (2005) 357-381.

[32] P. Pedregal, Optimal design in two-dimensional conductivity for a general cost depending on the field. Arch. Rational Mech. Anal. 182 (2006) 367-385.

[33] P. Pedregal, Div-Curl Young measures and optimal design in any dimension. Rev. Mat. Comp. 20 (2007) $239-255$.

[34] L. Tartar, An introduction to the Homogenization method in optimal design, in Lecture Notes in Mathematics 1740, A. Cellina and A. Ornelas Eds., Springer, Berlin/Heidelberg (2000) 47-156. 